\title{
Chapter 10. Optical Properties of Graphene in External Fields
}

\author{
Y. H. Chiu ${ }^{1, a}$, Y. C. Ou ${ }^{2, b}$, and M. F. $\operatorname{Lin}^{2, c}$ \\ ${ }^{1}$ National Center for Theoretical Sciences, Taiwan \\ ${ }^{2}$ Department of Physics, National Cheng Kung University, Tainan, Taiwan \\ ${ }^{a}$ e-mail address: airegg.py90g@nctu.edu.tw \\ ${ }^{b}$ e-mail address: 12897113@mail.ncku.edu.tw \\ ${ }^{c}$ e-mail address: mflin@mail.ncku.edu.tw
}

March 31, 2015

\begin{abstract}
The generalized tight-binding model, with the exact diagonalization method, is developed to investigate optical properties of graphene in five kinds of external fields. The quite large Hamiltonian matrix is transferred into the band-like one by the rearrangement of many basis functions; furthermore, the spatial distributions of wave functions on distinct sublattices are utilized to largely reduce the numerical computation time. The external fields have a strong influence on the number, intensity, frequency and structure of absorption peaks, and the selection rules. The optical spectra in a uniform magnetic field exhibit plentiful symmetric absorption peaks and obey a specific selection rule. However, there are many asymmetric peaks and extra selection rules under the modulated electric field, the modulated magnetic field, the composite electric and magnetic fields, and the composite magnetic fields.
\end{abstract}




\section{Introduction}

Monolayer graphene (MG), constructed from a single layer of carbon atoms densely packed in hexagonal lattice, was successfully produced by mechanical exfoliation.[1,2] This particular material offers an excellent system for studying two-dimensional (2D) physical properties, such as the quantum Hall effects, $3[15]$ and these properties could be preliminarily comprehended by the energy dispersion (or called energy band structure), which can directly reflect the main features of electronic properties. In the low-energy region of $\left|E^{c, v}\right| \leq 1 \mathrm{eV}$, MG possesses isotropic linear bands crossing at the $\mathbf{K}\left(\mathbf{K}^{\prime}\right)$ point and is regarded as a $2 \mathrm{D}$ zero-gap semiconductor, where $c(v)$ indicates the conduction (valence) bands. [16] The linear bands are symmetric about the Fermi level $\left(E_{F}=0\right)$ and become nonlinear and anisotropic with $\left|E^{c, v}\right|>1 \mathrm{eV} .[16]$ Most importantly, the quasiparticles related to the linear bands can be described by a Dirac-like Hamiltonian, [17] which is associated with relativistic particles and dominates the low-energy physical properties. 15, 18, 19, Such a special electronic structure has been verified by experimental measurements. [2, 20]

MG has become a potential candidate of nano-devices due to its exotic electronic properties. Well understanding the behavior of MG under external fields is useful for improving the characteristics of graphene-based nano-devices. Five cases of external fields (see table below), which can be experimentally produced, 21,27 are often applied to investigate the physical properties of few-layer graphenes (FLGs). In the presence of a uniform magnetic field (UM), the electronic states corresponding to the linear bands change into Landau levels (LLs) which obey a specific relationship $E^{c, v} \propto \sqrt{n^{c, v} B_{U M}}$, where $n^{c}\left(n^{v}\right)$ is the quantum number of the conduction (valence) states and $B_{U M}$ the magnetic field strength. The related anomalous quantum Hall effects and particular optical excitations have been verified experimentally. 14, 15] For a modulated magnetic field (MM), quasi-Landau levels (QLLs) possessing anisotropic behavior and the related optical absorption spectra with specified

selection rules were shown. 28, 29] Furthermore, Haldane predicted that MG in the modu- 
lated magnetic field could reveal quantum Hall effects even without any net magnetic flux through the whole space.13] Concerning a modulated electric potential (ME), the linear dispersions become oscillatory and extra Dirac cones are induced by the potential. $30-35$ Such a potential changes MG from a zero-gap semiconductor into a semimetal [33, 35 and makes MG exhibit Klein paradox effect associated with the Dirac cones. 17] For two cases of composite fields, a uniform magnetic field combined with a modulated magnetic field (UM-MM) and a uniform magnetic field combined with a modulated electric potential (UM-ME), the LL properties are drastically changed by the modulated fields. For both composite field cases, an unusual oscillation [36 40$]$ of the density of states (DOS) similar to the Weiss oscillation obtained in 2D electron gas (2DEG) were shown. Furthermore, the broken symmetry, displacement of the center location, and alteration of the amplitude strength of the LL wave functions were also obtained. 41, 42

Graphene-related systems are predicted to exhibit rich optical absorption spectra. The spectral intensity of MG is proportional to the frequency, but no prominent peak exists at $\omega<5 \mathrm{eV}$. 43 In FLGs, the interlayer atomic interactions drastically alter the two linear energy bands intersecting at $E_{F}=0.44 \sqrt{44}$ As a result, conspicuous absorption peaks arise in optical spectra, [55] where the peak structure, intensity and frequency are dominated by the layer number and the stacking configuration. Furthermore, under an external perpendicular electric field or a uniform perpendicular magnetic field, the main features of the optical properties of the FLGs are strongly modified. 49,56, 57] For theoretical studies, the complexity of calculating the optical absorption spectra is solved by the gradient approximation based on the generalized tight-binding model with exact-diagonalization method or effective-mass approximation. The way in which one can control the absorption peaks and selection rules is worthy to be reviewed in detail.

On the other hand, there has been a considerable amount of experimental research on graphene-related systems under a uniform perpendicular magnetic field. From the measured results, the features of MG and bilayer graphene are reflected in the magneto-optical 
spectra. 58, 59 That is to say, the LL energies are proportional to $\sqrt{n^{c, v} B_{0}}$ or $n^{c, v} B_{0}$. For any graphene system, the selection rule coming from the LLs close to $E_{F}=0$ is $\left.\Delta n=\left|n^{c}-n^{v}\right|=1.60-62\right]$ Moreover, similar results may also be found in AB-stacked graphite.62] However, experimental measurements on optical properties under a nonuniform or composite fields are not available so far.

In this chapter, we would like to focus on the optical absorption spectra of monolayer graphene under the five cases of external fields, UM, MM, ME, UM-MM and UM-ME cases. The tight-binding (TB) model with exact diagonalization method is introduced to solve the energy dispersions and then the gradient approximation is applied to obtain the optical absorption spectra. The main features of electronic properties, which include energy dispersions and wave functions, will be shown to comprehend the optical absorption properties, where the dependence of absorption frequency on external fields, optical selection rules and anisotropic behavior will be discussed in detail. In Sec. 10.1, the tight-binding model corresponding to the five cases of external fields is shown. In Secs. 10.2 to 10.6, the optical absorption spectra of MG under the UM, MM, ME, UM-MM and UM-ME cases will be reviewed, respectively. Finally, concluding remarks are presented in Sec. 10.7. 


\begin{tabular}{|l|l|}
\hline \multicolumn{2}{|c|}{ Physical properties of graphene under external fields } \\
\hline External fields & Related physical properties \\
\hline Uniform magnetic field & $\begin{array}{l}\text { Landau level and Abnormal quantum } \\
\text { Hall effect, [3] 15] Magneto-optical selection }\end{array}$ \\
\hline rule[58,59]
\end{tabular}




\section{Tight-Binding Model with Exact Diagonalization}

The low-frequency optical properties of graphene are determined by the $\pi$-electronic structure due to the $2 p_{z}$ orbitals of carbon atoms. The generalized tight-binding model with exact diagonalization method is developed to characterize the electronic properties and then the gradient approximation is applied to obtain the optical-absorption spectra. In the absence of external fields, there are two carbon atoms, the $a$ and $b$ atoms, in a primitive unit cell of MG, as shown in Fig. 10.1(a) by the green shadow, where the $x$ - and $y$-direction are respectively the armchair and zigzag directions of MG. This indicates that the Bloch wave function $\Psi$ is a linear superposition of two TB functions associated with the $2 p_{z}$ orbitals and expressed as $\Psi=\varphi_{a} \pm \varphi_{b}$, where $\varphi_{a}$ and $\varphi_{b}$ respectively stand for the tight-binding functions of the $a$ and $b$ atoms and are represented as 16

$$
\begin{aligned}
\varphi_{a} & =\sum_{a} \exp \left(i \mathbf{k} \cdot \mathbf{R}_{a}\right) \chi\left(\mathbf{r}-\mathbf{R}_{a}\right), \\
\varphi_{b} & =\sum_{b} \exp \left(i \mathbf{k} \cdot \mathbf{R}_{b}\right) \chi\left(\mathbf{r}-\mathbf{R}_{b}\right) .
\end{aligned}
$$

$\chi(\mathbf{r})$ is the normalized orbital $2 p_{z}$ wave function for an isolated atom. Moreover, the symbols $\gamma_{0}(=2.5 \mathrm{eV})$ and $b^{\prime}(=1.42 \AA)$ shown in Fig. 10.1(a) represent the nearest-neighbor atomic interaction (or called hopping integral) and the $\mathrm{C}-\mathrm{C}$ bond length, respectively. [16] Throughout this chapter, only $\gamma_{0}$ is taken into account and other atomic interactions are neglected.

In the presence of an external field, the primitive unit cell is no longer the one shown in Fig. 10.1(a) since the external field leads to a new periodic condition. Here we choose the rectangular unit cell marked by the green rectangle in Fig. 10.1(b) as the primitive unit cell of graphene under the five kinds of external fields, where $R=R_{U M}, R_{M M}, R_{M E}$, and $R_{C}$ (defined in the following) describe respectively the periods resulting from the uniform magnetic field, modulated magnetic field, modulated electric potential, and composite field. The major discussions are focused on $R$ along the armchair direction. Consequently, an 
(a)

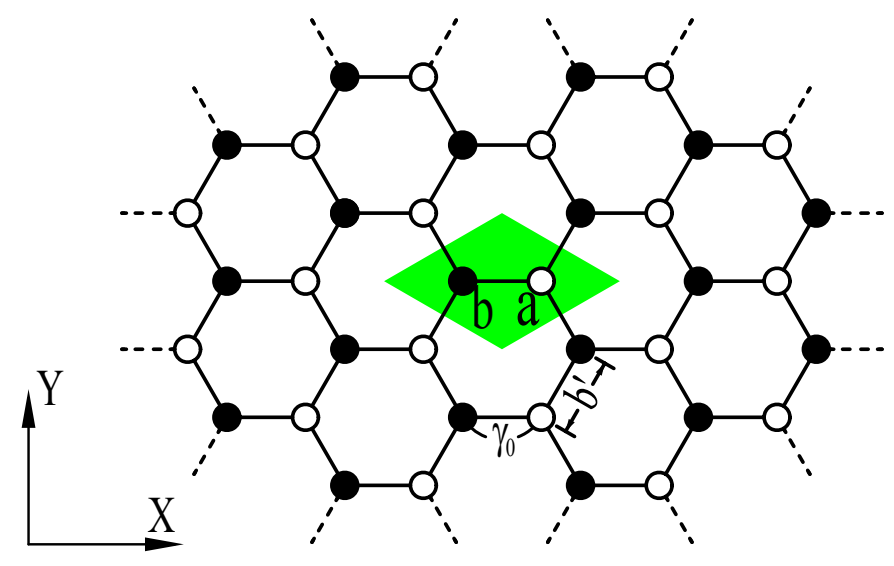

(b)
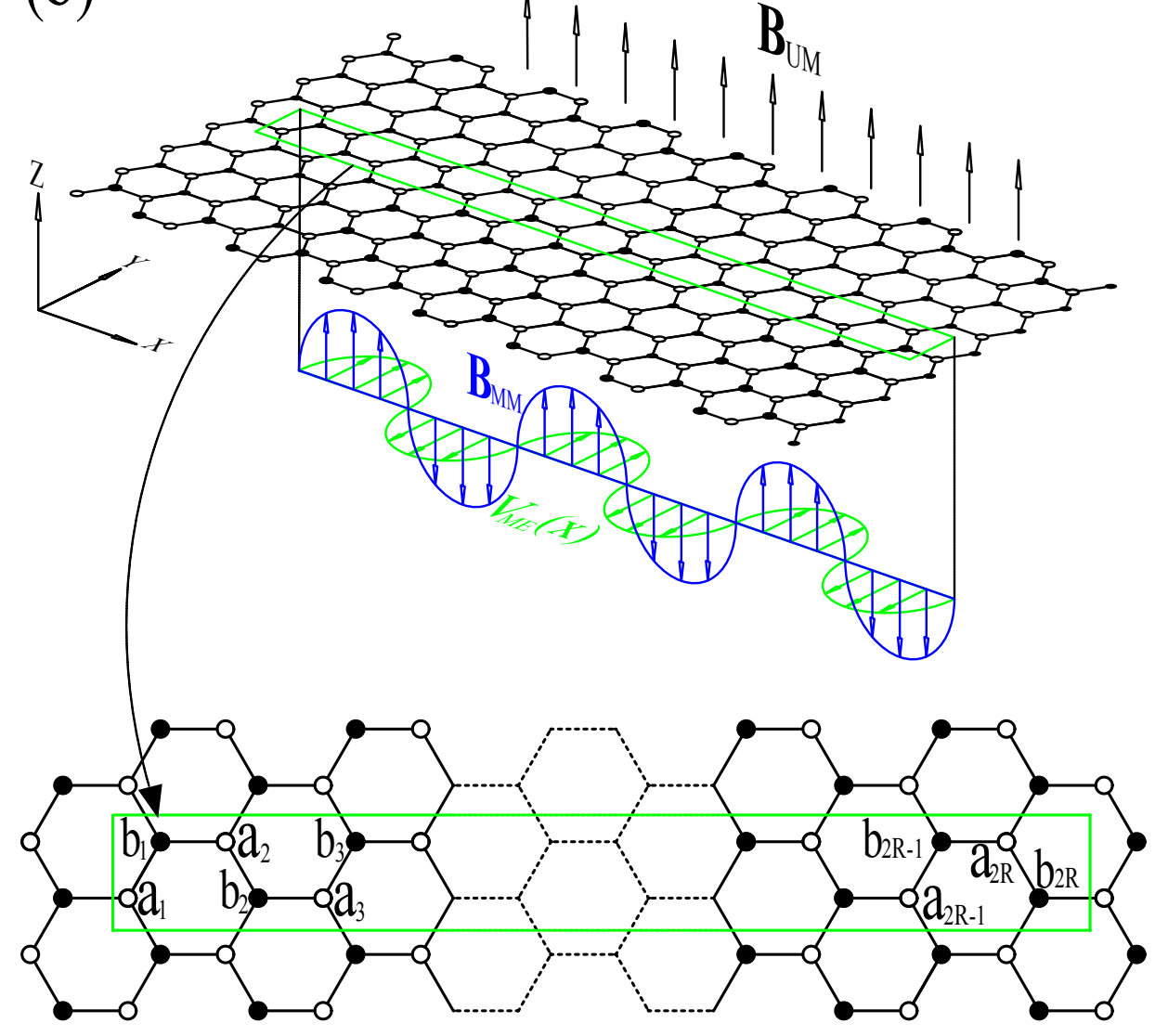

Figure 10.1. The primitive unit cell of monolayer graphene (a) in the absence and (b) in the presence of external fields. 
enlarged rectangular unit cell induced by an external field encompasses $2 R$ a atoms and $2 R$ $b$ atoms. This implies that $R$ determines the dimension of the Hamiltonian matrix, which is a $4 R \times 4 R$ Hermitian matrix spanned by $4 R$ TB functions associated with the $2 R a$ atoms and $2 R b$ atoms. Based on the arrangement of odd and even atoms in the primitive cell, the Bloch wave function $\left|\Psi_{\mathbf{k}}\right\rangle$ can have the expression:

$$
\left|\Psi_{\mathbf{k}}\right\rangle=\sum_{m=1}^{2 R-1}\left(A_{\mathbf{o}}^{c, v}\left|a_{m \mathbf{k}}\right\rangle+B_{\mathbf{o}}^{c, v}\left|b_{m \mathbf{k}}\right\rangle\right)+\sum_{m=2}^{2 R}\left(A_{\mathbf{e}}^{c, v}\left|a_{m \mathbf{k}}\right\rangle+B_{\mathbf{e}}^{c, v}\left|b_{m \mathbf{k}}\right\rangle\right) .
$$

$\left|a_{m \mathbf{k}}\right\rangle\left(\left|b_{m \mathbf{k}}\right\rangle\right)$ is the TB function corresponding to the $2 p_{z}$ orbital of the $m$ th $a(b)$ atom. $A_{\mathbf{o}}^{c, v}$ $\left(A_{\mathbf{e}}^{c, v}\right)$ and $B_{\mathbf{o}}^{c, v}\left(B_{\mathbf{e}}^{c, v}\right)$ are the subenvelope functions standing for the amplitudes of the wave functions of the $a$ - and $b$-atoms respectively, where $o(e)$ represents an odd (even) integer. Since the features of $A_{o}^{c, v}\left(B_{o}^{c, v}\right)$ and $A_{e}^{c, v}\left(B_{e}^{c, v}\right)$ are similar, choosing only the amplitudes $A_{\mathbf{o}}^{c, v}$ and $B_{\mathbf{o}}^{c, v}$ is sufficient to comprehend the electronic and optical properties we would like to discuss in this chapter. The $4 R \times 4 R$ Hamiltonian matrix, which determines the magneto-electronic properties, is a giant Hermitian matrix for the external fields actually used in experiments. To make the calculations more efficient, the matrix is transformed into an $M \times 4 R$ band-like matrix by a suitable rearrangement of the tight-binding functions, where $M$ is much smaller than $4 R$. For example, one can arrange the basis functions as the sequence: $\left|a_{1 \mathbf{k}}\right\rangle,\left|b_{2 R \mathbf{k}}\right\rangle,\left|b_{1 \mathbf{k}}\right\rangle,\left|a_{2 R \mathbf{k}}\right\rangle,\left|a_{2 \mathbf{k}}\right\rangle,\left|b_{2 R-1 \mathbf{k}}\right\rangle,\left|b_{2 \mathbf{k}}\right\rangle,\left|a_{2 R-1 \mathbf{k}}\right\rangle, \ldots \ldots\left|a_{R-1 \mathbf{k}}\right\rangle$, $\left|b_{R+2 \mathbf{k}}\right\rangle,\left|b_{R-1 \mathbf{k}}\right\rangle,\left|a_{R+2 \mathbf{k}}\right\rangle,\left|a_{R \mathbf{k}}\right\rangle,\left|b_{R+1 \mathbf{k}}\right\rangle,\left|b_{R \mathbf{k}}\right\rangle ;\left|a_{R+1 \mathbf{k}}\right\rangle$. Furthermore, distributions of the subenvelope functions are used to reduce the numerical computation time. The exact diagonalization method for numerical calculations is applicable to many kinds of magnetic, electric and composite fields.

For the UM case $\mathbf{B}_{U M}=B_{U M} \widehat{z}$, a Peierls phase $[28,63,65]$ related to the vector potential $\mathbf{A}_{U M}=B_{U M} x \widehat{y}$ is introduced in the TB functions. The phase difference between two lattice vectors $\left(\mathbf{R}_{m}\right.$ and $\left.\mathbf{R}_{m^{\prime}}\right)$ is defined as $G_{U M} \equiv \frac{2 \pi}{\phi_{0}} \int_{\mathbf{R}_{m^{\prime}}}^{\mathbf{R}_{m}} \mathbf{A}_{U M} \cdot d \mathbf{r}$, where $\phi_{0}=h c / e=$ $4.1356 \times 10^{-15}\left[\mathrm{~T} \mathrm{~m}^{2}\right]$ is the flux quantum. The Peierls phase periodic along the armchair direction provides a specific period set as $R_{U M}=\frac{\phi_{0} /\left(3 \sqrt{3} b^{2} / 2\right)}{B_{U M}}$ and the related Hamiltonian 
is a $4 R_{U M} \times 4 R_{U M}$ Hermitian matrix. The site energies, the diagonal matrix elements $\left\langle a_{m \mathbf{k}}|H| a_{m \mathbf{k}}\right\rangle$ and $\left\langle b_{m \mathbf{k}}|H| b_{m \mathbf{k}}\right\rangle$, are set to zero and the nonzero matrix elements related to $\gamma_{0}$ can be formulated as

$$
\left\langle b_{m \mathbf{k}}|H| a_{m^{\prime} \mathbf{k}}\right\rangle=\gamma_{0} \exp i\left[\mathbf{k} \cdot\left(\mathbf{R}_{m}-\mathbf{R}_{m^{\prime}}\right)+G_{U M}\right]
$$

Two kinds of periodic modulation fields along the armchair direction, the MM and ME cases, which can drastically change the physical properties of MG, are often selected for a study. For the MM case, $\mathbf{B}_{M M}=B_{M M} \sin \left(2 \pi x / l_{M M}\right) \widehat{z}$ is exerted on MG along the armchair direction, where $B_{M M}$ is the field strength and $l_{M M}$ is the period length with the modulation period $R_{M M}=l_{M M} / 3 b^{\prime}$. The vector potential is chosen as $\mathbf{A}_{M M}=\left(-B_{M M} \frac{l_{B}}{2 \pi} \cos \left(2 \pi x / l_{M M}\right)\right) \widehat{y}$ and the corresponding Peirls phase is $G_{M M} \equiv \frac{2 \pi}{\phi_{0}}$ $\int_{\mathbf{R}_{m^{\prime}}}^{\mathbf{R}_{m}} \mathbf{A}_{M M} \cdot d \mathbf{r}$. Thus the Hamiltonian matrix elements, which are similar to those in Eq. (10.3), are represented as

$$
\left\langle b_{m \mathbf{k}}|H| a_{m^{\prime} \mathbf{k}}\right\rangle=\gamma_{0} \exp i\left[\mathbf{k} \cdot\left(\mathbf{R}_{m}-\mathbf{R}_{m^{\prime}}\right)+G_{M M}\right] .
$$

For the ME case, $V_{M E}(x)=V_{M E} \cos \left(2 \pi x / l_{M E}\right)$ along the armchair direction with the potential strength $V_{M E}$ and the period length $l_{M E}$ is taken into account. As the period is sufficiently large, the electric potential affects only the site energies but not the nearestneighbor hopping integral. As a result, the site energies become

$$
\begin{aligned}
\left\langle a_{m \mathbf{k}}|H| a_{m \mathbf{k}}\right\rangle & =V_{M E} \cos \left[(m-1) \pi / R_{M E}\right] \equiv V_{m} \\
\left\langle b_{m \mathbf{k}}|H| b_{m \mathbf{k}}\right\rangle & =V_{M E} \cos \left[(m-2 / 3) \pi / R_{M E}\right] \equiv V_{m+1 / 3}
\end{aligned}
$$

where $R_{M E}=l_{M E} / 3 b^{\prime}$ is the modulation period. The Hamiltonian matrices for the modulated magnetic field and the modulated electric potential are $4 R_{M M} \times 4 R_{M M}$ and $4 R_{M E} \times$ $4 R_{M E}$ Hermitian matrices, respectively.

For a composite field case, a new periodicity, which is associated with periods induced by a uniform magnetic field and a modulated field, has to be defined. The rectangular unit 
cell is enlarged along the $x$-direction and the dimensionality of the Hamiltonian matrix has to agree with the least common multiple of $R_{U M}$ and $R_{M M}\left(R_{U M}\right.$ and $\left.R_{M E}\right)$ for the UMMM (UM-ME) case, namely $R_{C}$. The rectangular unit cell corresponding to each composite field contains $4 R_{C}$ atoms $\left(2 R_{C}\right.$ a atoms and $2 R_{C} b$ atoms $)$, and the magneto-electronic wave functions are linear combinations of the $4 R_{C}$ TB functions. In a composite field case, the matrix elements are superposed by the elements associated with each combined external field. For the sake of convenience, we put the matrix elements in Eqs. (10.3)-(10.5) together as a common case and the elements are rewritten as

$$
\begin{aligned}
\left\langle b_{m \mathbf{k}}|H| a_{m^{\prime} \mathbf{k}}\right\rangle & =\gamma_{0} \exp i\left[\mathbf{k} \cdot\left(\mathbf{R}_{m}-\mathbf{R}_{m^{\prime}}\right)+G_{U M}+G_{M M}\right] \\
\left\langle a_{m \mathbf{k}}|H| a_{m \mathbf{k}}\right\rangle & =V_{m} \\
\left\langle b_{m \mathbf{k}}|H| b_{m \mathbf{k}}\right\rangle & =V_{m+1 / 3} .
\end{aligned}
$$

The off-diagonal elements are associated with the Peierls phases induced by the magnetic fields and the diagonal elements are related to the site energies induced by the modulated electric field. By diagonalizing the matrix, the energy dispersion $E^{c, v}$ and the wave function $\Psi^{c, v}$ are obtained. It should be noted that the $k_{x}$-dependent dispersions can be ignored when the period $R$ is sufficiently large and thus only $k_{y}$-dependent dispersions are shown for the following discussions.

When a monolayer graphene is excited from the occupied valence to the unoccupied conduction bands by an electromagnetic field, only inter- $\pi$-band excitations exist at zero temperature. Based on the Fermi's golden rule, the optical absorption function results in the following form

$$
\begin{aligned}
A(\omega) \propto & \sum_{c, v, \widetilde{n}, \widetilde{n}^{\prime}} \int_{1 s t B Z} \frac{d \mathbf{k}}{(2 \pi)^{2}}\left|\left\langle\Psi^{c}(\mathbf{k}, n)\left|\frac{\widehat{\mathbf{E}} \cdot \mathbf{P}}{m_{e}}\right| \Psi^{v}\left(\mathbf{k}, n^{\prime}\right)\right\rangle\right|^{2} \\
& \times \operatorname{Im}\left[\frac{f\left(E^{c}(\mathbf{k}, n)\right)-f\left(E^{v}\left(\mathbf{k}, n^{\prime}\right)\right)}{E^{c}(\mathbf{k}, n)-E^{v}\left(\mathbf{k}, n^{\prime}\right)-\omega-i \Gamma}\right]
\end{aligned}
$$

where $f(E(\mathbf{k}, \tilde{n}))$ is the Fermi-Dirac distribution function, and $\Gamma\left(=2 \times 10^{-4} \gamma_{0}\right)$ is the broadening parameter. The electric polarization $\widehat{\mathbf{E}}$ is the unit vector of an electric po- 
larization. Results for $\widehat{\mathbf{E}}$ along the armchair and zigzag directions are taken into account for discussions. Within the gradient approximation, $66[68$ the velocity matrix element $M^{c v}=\left\langle\Psi^{c}(\mathbf{k}, \widetilde{n})\left|\frac{\widehat{\mathbf{E}} \cdot \mathbf{P}}{m_{e}}\right| \Psi^{v}\left(\mathbf{k}, \widetilde{n}^{\prime}\right)\right\rangle$ is formulated as

$$
\sum_{m, m /=1}^{2 R_{C}}\left(A^{c *} \times B^{v}\right) \nabla_{\mathbf{k}}\left\langle a_{m \mathbf{k}}|H| b_{m^{\prime} \mathbf{k}}\right\rangle+h . c . .
$$

Equation (10.8) implies that the main features of the wave functions are major factors in determining the selection rules and the absorption rate of the optical excitations. Similar gradient approximations have been successfully applied to explain optical spectra of carbon-related systems, e.g., graphite, [69] graphite intercalation compounds, [70] carbon nanotubes, [71] few-layer graphenes, [72] and graphene nanoribbons. [22] 


\section{Uniform Magnetic Field}

\subsection{Landau Level Spectra}

In this section, we mainly focus on drastic changes of the Dirac cone as the result of a uniform perpendicular magnetic field. The magnetic field causes the states to congregate and induces dispersionless Landau levels, as shown in Fig. 10.2(a) for $B_{U M}=5 \mathrm{~T}$ at $k_{x}=0$. The unoccupied LLs and occupied LLs are symmetric about the Fermi level $\left(E_{F}=0\right)$. Each LL is characterized by the quantum number $n^{c, v}$, which corresponds to the the number of zeros in the eigenvectors of harmonic oscillator. 50, 53] Each LL is fourfold degenerate without considering the spin degeneracy. Its energy may be approximated by a simple square-root relationship $\left|E_{n}^{c, v}\right| \propto \sqrt{n^{c, v} B_{U M}}, 12,74 \mid$ which is valid only for the range of $\left|E_{n}^{c, v}\right| \leq \pm 1 \mathrm{eV} \cdot[12$

\subsection{Landau Level Wave Functions}

The LL wave functions, as shown in Figs. 10.2(b) and 10.2(c), exhibit the versatility of spatial symmetry and can be described by the eigenvectors $\left(\varphi_{n}(x)\right)$ of harmonic oscillator, which obey the relationships, $\left\langle\varphi_{n}(x) \mid \varphi_{n^{\prime}}(x)\right\rangle=\delta_{n, n \prime}$ and $\varphi_{n}(x)=0$ for $n<0$. The wave functions are distributed around the localization center, that is at the $5 / 6$ position of the enlarged unit cell. Similar localization centers corresponding to the other degenerate states occur at the $1 / 6,2 / 6$, and $4 / 6$ positions. The subenvelope functions can be expressed as

$$
\begin{aligned}
& A_{o, e}^{c, v} \propto \varphi_{n^{c, v}}\left(x_{1}\right) \pm \varphi_{n^{c, v}-1}\left(x_{2}\right), B_{o, e}^{c, v} \propto \varphi_{n^{c, v}-1}\left(x_{1}\right) \mp \varphi_{n^{c, v}}\left(x_{2}\right), \\
& A_{o, e}^{c, v} \propto \varphi_{n^{c, v}-1}\left(x_{3}\right) \pm \varphi_{n^{c, v}}\left(x_{4}\right), B_{o, e}^{c, v} \propto \varphi_{n^{c, v}}\left(x_{3}\right) \mp \varphi_{n^{c, v}-1}\left(x_{4}\right), \\
& \text { for } x_{1}=1 / 6, x_{2}=5 / 6, x_{3}=2 / 6, \text { and } x_{4}=4 / 6 .
\end{aligned}
$$

However, it is adequate to only consider any one center in evaluating the absorption spectra due to their identical optical responses. 


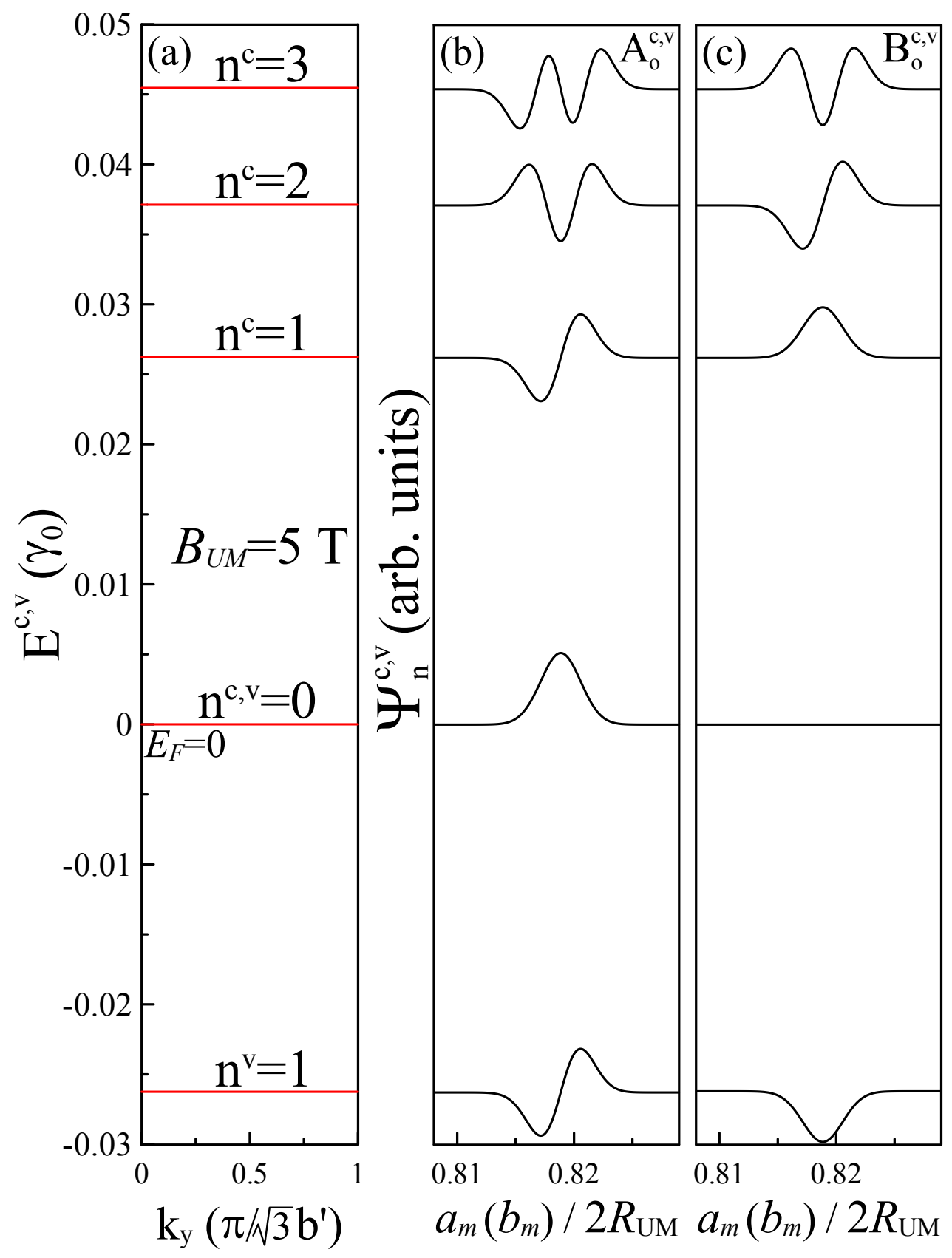

Figure 10.2. The Landau level spectrum for the uniform magnetic field $B_{U M}=5 \mathrm{~T}$. The Landau level wave functions corresponding to (b) the $a$ - and (c) the $b$-atoms. 

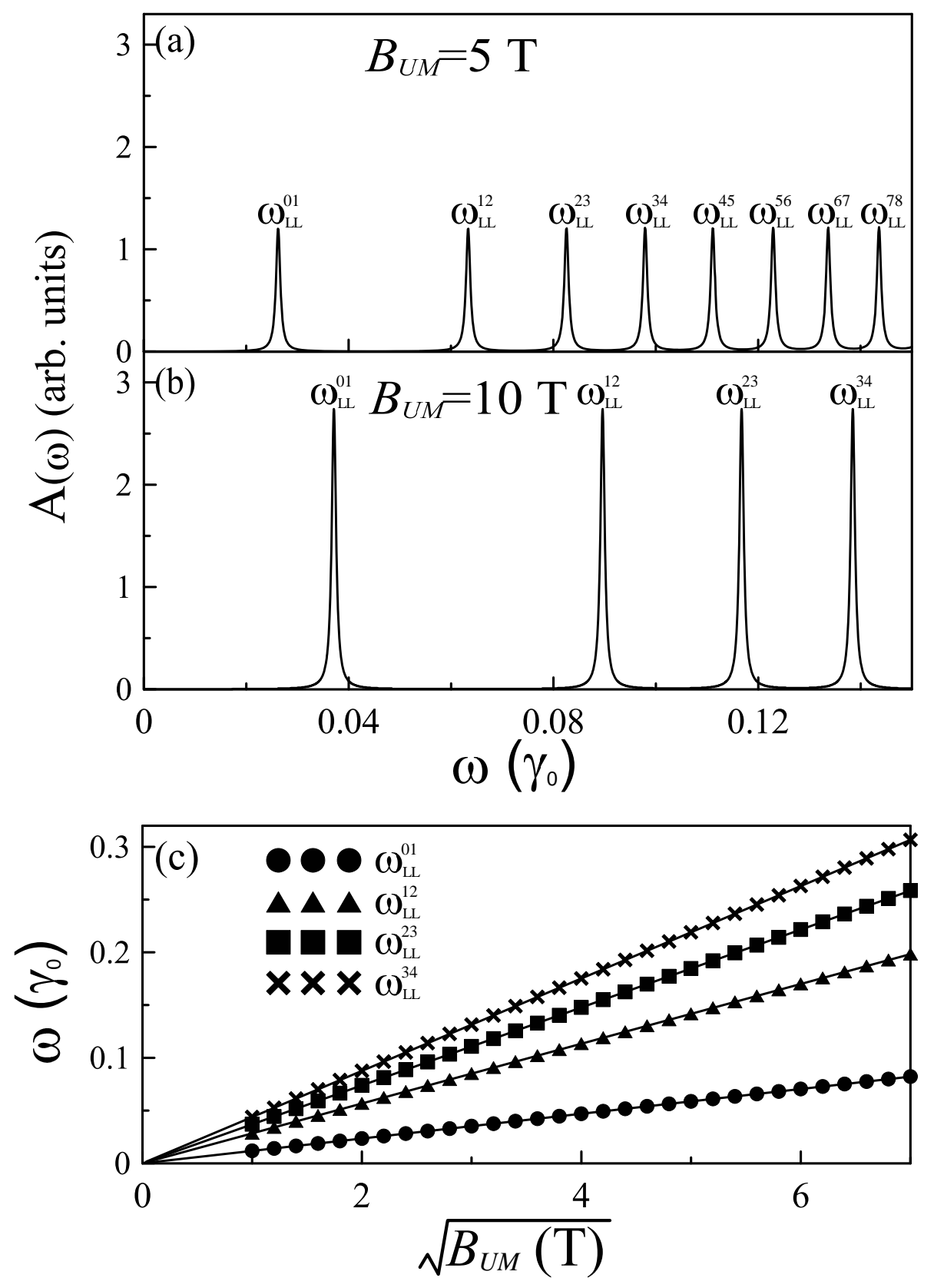

Figure 10.3. The optical absorption spectra for (a) $B_{U M}=5 \mathrm{~T}$ and (b) $B_{U M}=10 \mathrm{~T}$. (c) The dependence of the absorption frequency on the square root of field strength $B_{U M}$. 


\subsection{Optical Absorption Spectra of Landau Levels}

The low-frequency optical absorption spectrum of the LLs presents many interesting features as shown in Fig. 10.3(a) for $B_{U M}=5 \mathrm{~T}$. The spectrum exhibits many delta-functionlike symmetric peaks with a uniform intensity. Such peaks suggest that LLs possess a zero-dimensional (0D) band structure or density of states. The optical transition channel with respect to each absorption peak can be clearly identified. A single peak $\omega_{L L}^{n n^{\prime}}$ is generated by two transition channels $n^{\prime} \mathrm{LL} \rightarrow n \mathrm{LL}$ and $n \mathrm{LL} \rightarrow n^{\prime} \mathrm{LL}$, where the symbol $n^{\prime} \rightarrow n$ is used, for the sake of convenience, to represent the transition from the valence states with $n^{\prime}$ to the conduction states with $n$ throughout this chapter. The quantum numbers related to the LL transitions must satisfy a specific selection rule, i.e., $\Delta n=\left|n^{c}-n^{v}\right|=1$. The selection rule is established by the main features of the wave functions. The velocity matrix $M^{c v}$, a dominant factor for the excitations of the prominent peaks, strongly depends on the number of zeros of $A_{\mathbf{o}}^{c, v}$ and $B_{\mathbf{o}}^{c, v}$. It has non-zero values only when $A_{\mathbf{o}}^{c(v)}$ and $B_{\mathbf{o}}^{v(c)}$, expressed in orthogonality of $\varphi_{n}(x)$, possess the same number of zeros. Moreover, examining all the transitions reveals the following relationship: $A_{\mathbf{o}}^{c, v}\left(n^{c, v}\right) \propto B_{\mathbf{o}}^{c, v}\left(n^{c, v}+1\right)$, with $A_{\mathbf{o}}^{c}=A_{\mathbf{o}}^{v}$ and $B_{\mathbf{o}}^{c}=-B_{\mathbf{o}}^{v}$. In other words, the quantum numbers of the conduction and valence LLs differ by one when $A_{\mathbf{o}}^{c(v)}$ and $B_{\mathbf{o}}^{v(c)}$ have the same $\varphi_{n}(x)$.

In addition to the optical selection rules, the peak intensity and absorption frequency also deserve a discussion. In Fig. 10.3(b), the peak intensity is strengthened, whereas the peak number is reduced as the field strength increases. This is a result of the high degree of degeneracy in the first Brillouin zone and the expanded energy spacing between the LLs. The field-dependent absorption frequencies of the first four peaks $\omega_{L L}^{01}, \omega_{L L}^{12}, \omega_{L L}^{23}$, and $\omega_{L L}^{34}$ are shown in Fig. 10.3(c). The frequencies become much higher in a stronger field. There exists a special square-root relation between $\omega_{L L}^{n n^{\prime}}$ and $B_{0}$, i.e., $\omega_{L L}^{n n^{\prime}} \propto \sqrt{B_{U M}}$, which has been confirmed by magneto-optical spectroscopy methods, such as experimental measurements of the absorption coefficient, 75,76$]$ cyclotron resonance, 77,79$]$ and quantum 
Hall conductivity. 2, 80, 82 This square-root relation only exists in the lower frequency range $\omega<0.4 \gamma_{0}(\sim 1 \mathrm{eV})$. In the higher frequency range, LLs are too densely packed to be separated from one another.[12] This leads to the disappearance of the relation between $\omega_{L L}^{n n^{\prime}}$ and $B_{U M}$ 


\section{Spatially Modulated Magnetic Field}

\subsection{Quasi-Landau Level Spectra}

Compared with the uniform case, a modulated magnetic field has a different impact on the electronic properties and leads to the diverse features observed in the optical absorption spectra. The presence of a modulated field has multiple effects on the energy bands, as shown in Fig. 10.4 for $B_{M M}=10 \mathrm{~T}$ and $R_{M M}=500$. In the lower energy region, parabolic subbands appear around $k_{y}=k_{1}=2 / 3$. The conduction and valence subbands are symmetric about the Fermi level $\left(E_{F}=0\right)$. The subbands nearest to $E_{F}=0$ are partially flat and nondegenerate. The other parabolic subbands characterized by weak energy dispersions have double degeneracy and one original band-edge state at $k_{1}$. The modulation effects on parabolic energy subbands result in four extra band-edge states at the sites on both sides of $k_{1}$. They demonstrate the strongest dispersion and destruction of the double degeneracy. The low-energy subbands are regarded as quasi-Landau levels, which exhibit similar features of the LLs generated from a uniform magnetic field. Moreover, the $k_{y}$ range with respect to the weak dispersion and partial flat bands grows with increasing field strength and a longer modulation period. On the contrary, when the influence of the modulation field become much weaker with increasing energy, the parabolic subbands in the higher energy region are similar to the twofold degenerate subbands directly obtained from the zone folding of $M G$ in the $B_{M M}=0$ case (not shown).

\subsection{Quasi-Landau Level Wave Functions}

In the presence of a modulated magnetic field, the alterations of the wave functions are rather drastic. First, the QLL wave functions corresponding to $k_{1}$ are shown in Figs. 10.5(a)-(f). The wave functions are composed of two tight-binding functions centered at $x_{1}$ and $x_{2} . A_{\mathbf{o}}^{c}\left(B_{\mathbf{o}}^{c}\right)$ has two subenvelope functions $A_{\mathbf{o}}^{c}\left(x_{1}\right)\left(B_{\mathbf{o}}^{c}\left(x_{1}\right)\right)$ and $A_{\mathbf{o}}^{c}\left(x_{2}\right)\left(B_{\mathbf{o}}^{c}\left(x_{2}\right)\right)$ 


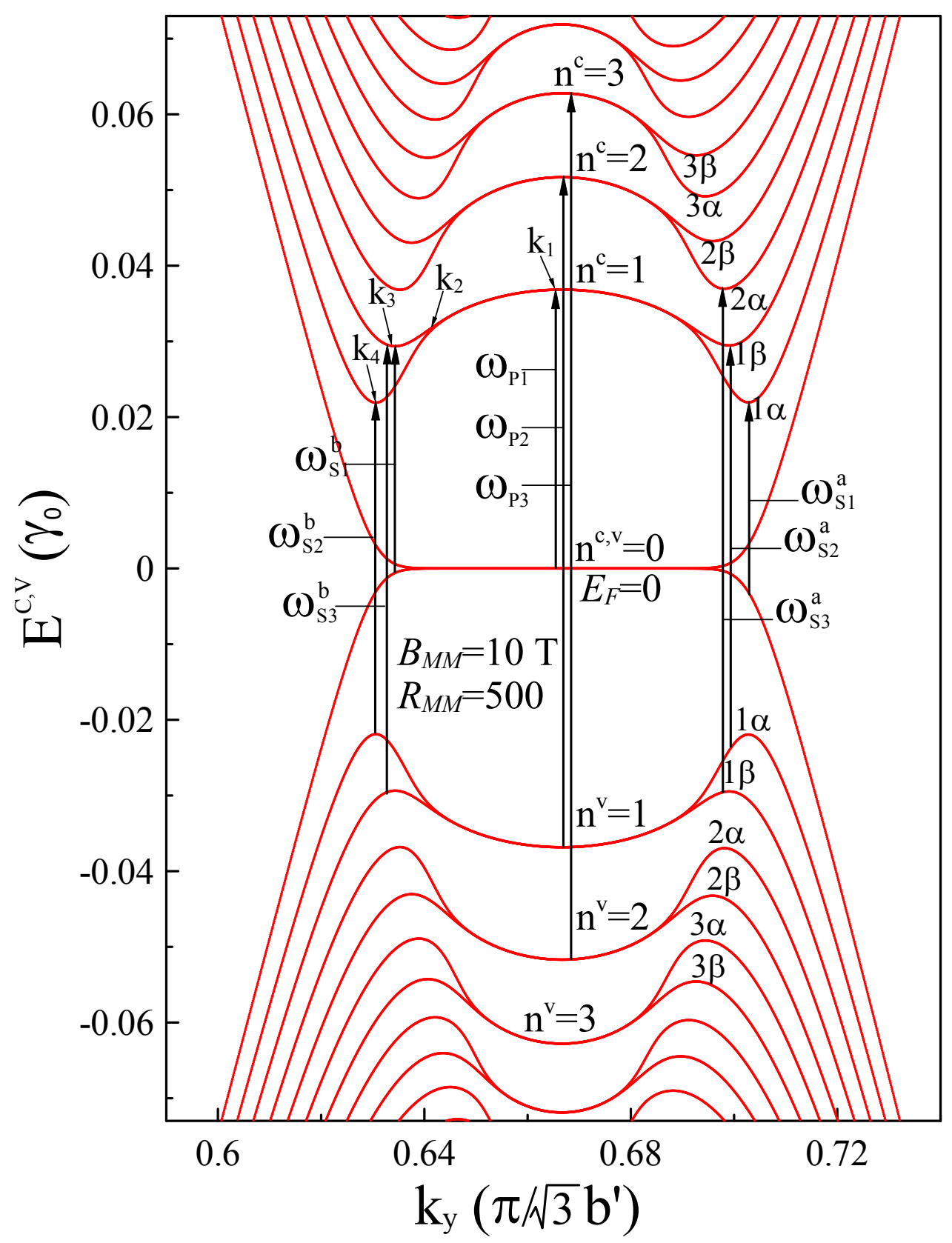

Figure 10.4. The energy dispersions and the illustration of optical excitation channels for the modulated magnetic field along the armchair direction with $R_{M M}=500$ and $B_{M M}=10$ $\mathrm{T}$. 
centered at $x_{1}=1 / 4$ and $x_{2}=3 / 4$ of the primitive unit cell, respectively. The positions $x_{1}$ and $x_{2}$ are located at where the field strength is at a maximum. The number of zeros of $A_{\mathbf{o}}^{c}\left(x_{2}\right)\left(B_{\mathbf{o}}^{c}\left(x_{1}\right)\right)$ is higher than that of $A_{\mathbf{o}}^{c}\left(x_{1}\right)\left(B_{\mathbf{o}}^{c}\left(x_{2}\right)\right)$ by one at each QLL. A similar behavior is also shown by the valence wave function, where only the sign is flipped in either $A_{\mathbf{o}}^{v}$ or $B_{\mathbf{o}}^{v}$. The effective quantum number $n^{c, v}$ is defined by the larger number of zeros of the subenvelope functions. In addition, the twofold degenerate QLLs have similar wave functions (black curves and red dashed curves), with the only difference in terms of the sign change in the subenvelope functions. The wave functions at $k_{1}$ can be expressed as

$$
\begin{aligned}
& \qquad \begin{array}{l}
A_{o, e}^{c} \propto \Psi_{n^{c}-1}\left(x_{1}\right) \pm \Psi_{n^{c}}\left(x_{2}\right), A_{o, e}^{v} \propto \Psi_{n^{v}-1}\left(x_{1}\right) \mp \Psi_{n^{v}}\left(x_{2}\right), \\
B_{o, e}^{c}
\end{array} \\
& \text { for } x_{1}=1 / 4 \text { and } x_{2}=3 / 4 .
\end{aligned}
$$

The wave functions would be strongly modified as the wave vectors gradually move away from $k_{1}$. Secondly, the wave functions at several special $k$ points are illustrated to examine the effects caused by the modulated magnetic field. As the wave vector moves to $k_{y}=k_{2}$, the doubly degenerate QLL starts to separate into two subbands. The two subenvelope functions $A_{\mathbf{o}}^{c}\left(x_{1}\right)\left(B_{\mathbf{o}}^{c}\left(x_{1}\right)\right)$ and $A_{\mathbf{o}}^{c}\left(x_{2}\right)\left(B_{\mathbf{o}}^{c}\left(x_{2}\right)\right)$ move toward each other and shift to the center of the primitive unit cell with nearly overlapping, as shown in Fig. 10.5(g) and 10.5(h). At $k_{3}$ and $k_{4}$, the higher and lower subbands have the extra band-edge states $1 \alpha$ and $1 \beta$, respectively. The subenvelope functions of the $1 \alpha$ state, as shown in Fig. 10.5(i) and $10.5(\mathrm{j})$, exhibit a strong overlapping behavior compared to those at $k_{y}=k_{2}$ (red-dashed curves in Fig. 10.5(g) and 10.5(h)). Similar behavior can also be found in the wave functions at $1 \beta$. This implies that there is a higher degree of overlap in the subenvelope functions at the extra band-edge states $n^{c, v} \alpha$ and $n^{c, v} \beta$. Moreover, the two states associated with the 
different linear combinations of $A_{\mathbf{o}}^{c}\left(x_{1}\right)\left(B_{\mathbf{o}}^{c}\left(x_{1}\right)\right)$ and $A_{\mathbf{o}}^{c}\left(x_{2}\right)\left(B_{\mathbf{o}}^{c}\left(x_{2}\right)\right)$ are represented as

$$
\begin{aligned}
& A_{o, e}^{c} \propto \Psi_{n^{c}-1}\left(x_{1}\right)+\Psi_{n^{c}}\left(x_{2}\right) \text { for } n^{c} \alpha \text { and } \Psi_{n^{c}-1}\left(x_{1}\right)-\Psi_{n^{c}}\left(x_{2}\right) \text { for } n^{c} \beta, \\
& B_{o, e}^{c} \propto \Psi_{n^{c}}\left(x_{1}\right)-\Psi_{n^{c}-1}\left(x_{2}\right) \text { for } n^{c} \alpha \text { and } \Psi_{n^{c}}\left(x_{1}\right)+\Psi_{n^{c}-1}\left(x_{2}\right) \text { for } n^{c} \beta, \\
& A_{o, e}^{v} \propto \Psi_{n^{v}-1}\left(x_{1}\right)-\Psi_{n^{v}}\left(x_{2}\right) \text { for } n^{v} \alpha \text { and } \Psi_{n^{v}-1}\left(x_{1}\right)+\Psi_{n^{v}}\left(x_{2}\right) \text { for } n^{v} \beta, \\
& B_{o, e}^{v} \propto \Psi_{n^{v}}\left(x_{1}\right)+\Psi_{n^{v}-1}\left(x_{2}\right) \text { for } n^{v} \alpha \text { and } \Psi_{n^{v}}\left(x_{1}\right)-\Psi_{n^{v}-1}\left(x_{2}\right) \text { for } n^{v} \beta, \\
& \text { for } x_{1} \approx x_{2} \simeq 1 / 2 .
\end{aligned}
$$

\subsection{Optical Absorption Spectra of Quasi-Landau Levels}

Under the modulated magnetic field, the parabolic energy bands possess several bandedge states. A wave function composed of two tight-binding functions presents a complex overlapping behavior. The above-mentioned main features of the electronic properties are expected to be directly reflected in optical excitations. The low-frequency optical absorption spectra for $R_{M M}=500$ and $B_{M M}=10 \mathrm{~T}$, as shown in Fig. 10.6(a) by the black

and blue solid curves for $\widehat{\mathbf{E}} \perp \widehat{x}$ and $\widehat{\mathbf{E}} \| \widehat{x}$ respectively, exhibit rich asymmetric peaks in the square-root divergent form. These peaks can be divided into the principal peaks $\omega_{P}$ 's and the subpeaks $\omega_{S}$ 's based on the optical excitations resulting from the original band-edge and extra band-edge states, respectively. $\omega_{S}$ 's can be further classified into two subgroups $\omega_{S}^{a}$ 's and $\omega_{S}^{b}$ 's which primarily come from the excitations of extra band-edge states $\alpha \rightarrow \beta$ $(\beta \rightarrow \alpha)$ and $\alpha \rightarrow \alpha(\beta \rightarrow \beta)$, respectively. What is worth mentioning is that the

spectra for $\widehat{\mathbf{E}} \perp \widehat{x}$ and $\widehat{\mathbf{E}} \| \widehat{x}$ are distinct, especially for the subpeaks $\omega_{S}$ 's. The former is mainly composed of the subgroup $\omega_{S}^{a}$, while the latter mainly consists of the subgroup $\omega_{S}^{b}$. This implies that the optical absorption spectra reflect the anisotropy of the polarization direction. For the modulation along the zigzag direction at $R_{M M}=866$ and $B_{M M}=10 \mathrm{~T}$, the absorption spectrum (red dashed curve in Fig. 10.6(a)) shows features similar to those of the spectrum corresponding to the armchair direction at $R_{M M}=500$ and $B_{M M}=10$ T. $R_{M M}=866$ for the zigzag direction and $R_{M M}=500$ for the armchair direction possess 


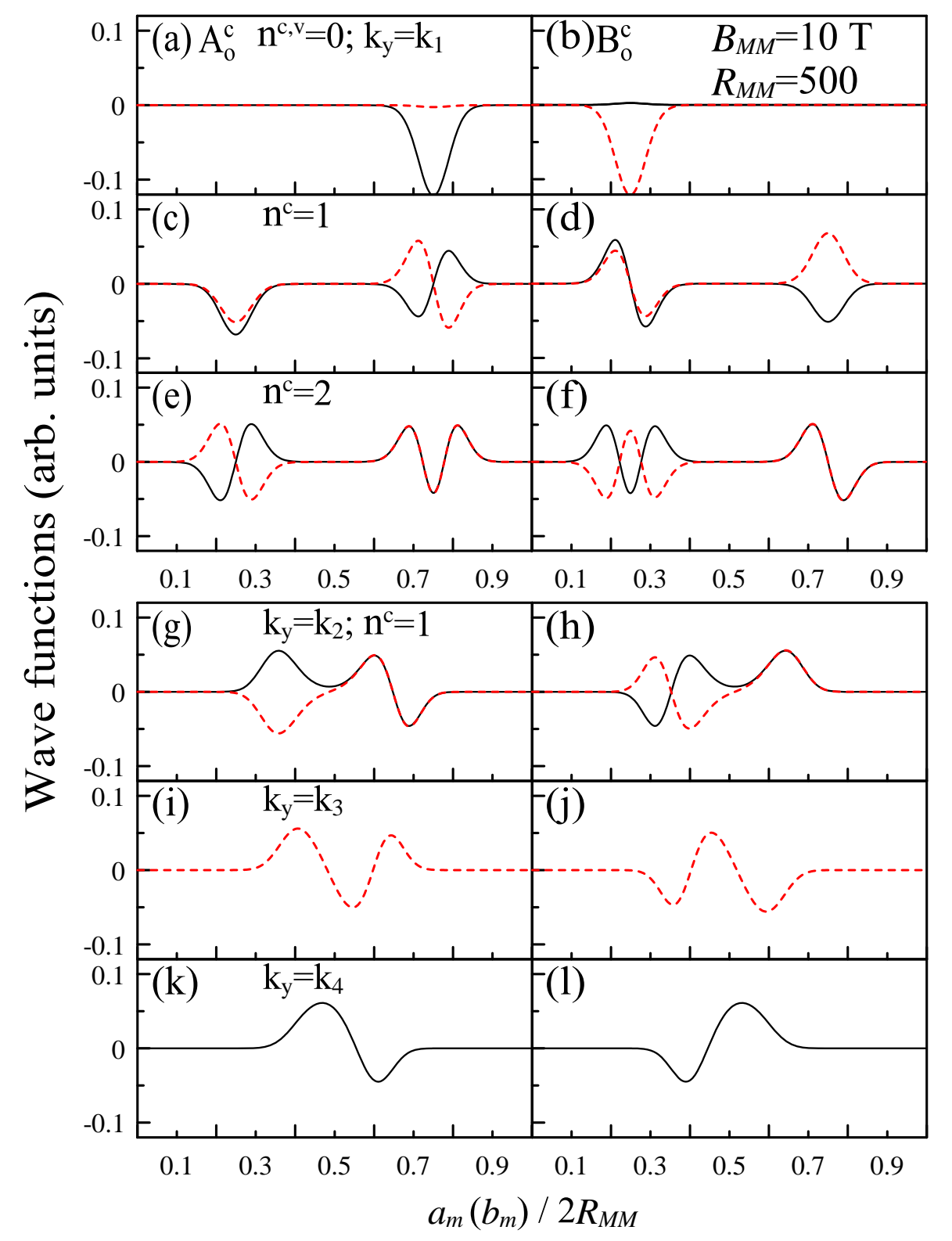

Figure 10.5. The wave functions of Quasi-Landau levels at (a)-(f) the original band-edge state $k_{1}$ with the quantum numbers $n^{c, v}=0, n^{c}=1$ and $n^{c}=2$, (g) and (h) the split point $k_{2}$ with $n^{c}=1$, and (i)-(l) two extra band-edge states $k_{3}$ and $k_{4}$ with $n^{c}=1$. 
the same period length based on the definitions $R_{M M}=l_{M M} / 3 b^{\prime}$ and $R_{M M}=l_{M M} / \sqrt{3} b^{\prime}$ associated with the zigzag and armchair directions, respectively. Moreover, the anisotropic features of the modulation directions will be revealed in the higher frequency region or the smaller modulation length.

As the field strength rises, the peak height and frequency of the principal peaks increase, and the peak number decreases, as shown in Fig. 10.6(b) by the red curve for $R_{M M}=500$ and $B_{M M}=20 \mathrm{~T}$. These results mean that the congregation of electronic states is more pronounced as the field strength grows. In addition to the field strength, the opticalabsorption spectrum is also influenced by the modulation period. In Fig. 10.6(b), the blue curve shows the optical spectra of $B_{M M}=10 \mathrm{~T}$ for $R_{M M}=1000$. The subpeaks strongly depend on the period, i.e., they represent different peak heights and frequencies with the variation of $R_{M M}$. However, the opposite is true for the principal peaks.

The peaks in the low-frequency absorption spectra can arise from the different selection rules. Fig. 10.4 illustrates the transition channels of the principal peaks resulting from the original band-edge states denoted as $\omega_{P n}$ 's in Fig. 10.6(a). Each $\omega_{P n}$ corresponds to the transition channels from QLLs $n \rightarrow n+1$ and $n \rightarrow n+1$ at the original band-edge state and the selection rule is represented by $\Delta n=\left|n^{c}-n^{v}\right|=1$ which is same as that related to LLs. The main reason for this is that the subenvelope functions $A_{\mathbf{o}}^{c(v)}\left(x_{1}\right)\left(A_{\mathbf{o}}^{c(v)}\left(x_{2}\right)\right)$ and $B_{\mathbf{o}}^{v(c)}\left(x_{1}\right)\left(B_{\mathbf{o}}^{v(c)}\left(x_{2}\right)\right)$ associated with the effective quantum numbers $n+1(n)$ and $n$ $(n+1)$ have the same number of zeros, respectively. As discussed in the former section, peaks arise in the optical absorption spectra when the number of zeros is the same for $A_{\mathbf{o}}^{c(v)}$ and $B_{\mathbf{o}}^{v(c)}$ in Eq. (10.10). The subpeaks originating from the extra band-edge states display a more complex behavior. The excitation channels for the subpeaks $\omega_{S n}^{a}$ and $\omega_{S n}^{b}$ in Fig. 10.6(a) are shown in Fig. 10.4. The subpeaks of different selection rules, $\Delta n=0$ and 1 , come into existence simultaneously. For example, $\omega_{S 2}^{a}$ comes from the excitation channel $1 \alpha \rightarrow 1 \beta(1 \beta \rightarrow 1 \alpha)$ and $\omega_{S 3}^{a}$ comes from the excitation channel $1 \beta \rightarrow 2 \alpha(2 \alpha \rightarrow 1 \beta)$. The extra selection rule $\Delta n=0$ reflects the overlap of subenvelope functions $A_{\mathbf{o}}^{c}\left(x_{1}\right)\left(B_{\mathbf{o}}^{c}\left(x_{1}\right)\right)$ 


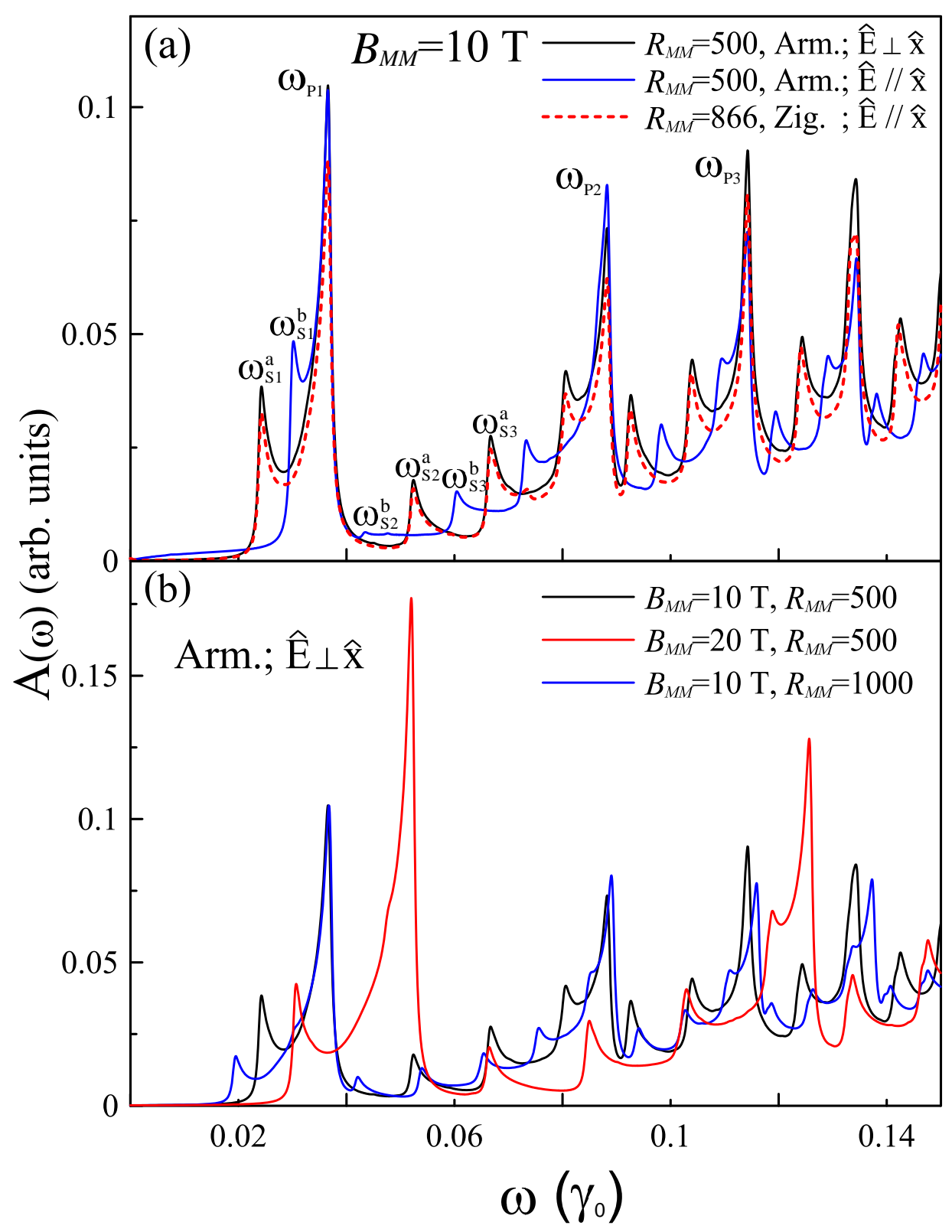

Figure 10.6. The optical absorption spectra for (a) $B_{M M}=10 \mathrm{~T}$ at a fixed periodic length with modulation and polarization along the armchair and zigzag directions and (b) different modulation periods and field strengths with both the modulation and polarization along the armchair direction. 
and $A_{\mathbf{o}}^{c}\left(x_{2}\right)\left(B_{\mathbf{o}}^{c}\left(x_{2}\right)\right)$ located around $x_{1} \approx x_{2} \approx 1 / 2$. The subenvelope functions $A_{\mathbf{o}}^{c(v)}\left(x_{1}\right)$ $\left(A_{\mathbf{o}}^{c(v)}\left(x_{2}\right)\right)$ and $B_{\mathbf{o}}^{v(c)}\left(x_{2}\right)\left(B_{\mathbf{o}}^{v(c)}\left(x_{1}\right)\right)$ of the effective quantum number $n$ also have the same number of zeros at the identical position, a cause leading to the extra selection rule $\Delta n=0$.

The frequency of principal peaks in the optical absorption spectra is worth a closer investigation. The relation between the frequencies of the first four principal peaks and the modulation period is shown in Fig. 10.7(a). The $\omega_{P}$ 's present a very weak dependence on the period as $R_{M M}$ becomes sufficiently large, whereas they exhibit a strong dependence on the field strength. The frequencies grow with increased $B_{M M}$, as shown in Fig. 10.7(b). The dependence of $\omega_{P}$ 's on $B_{M M}$ is similar to what is seen in the case of a uniform perpendicular magnetic field, i.e., $\omega_{P}$ 's $\propto \sqrt{B_{M M}}$, as indicated by the red lines. The predicted results could be verified by optical spectroscopy. $14,20,79$ 

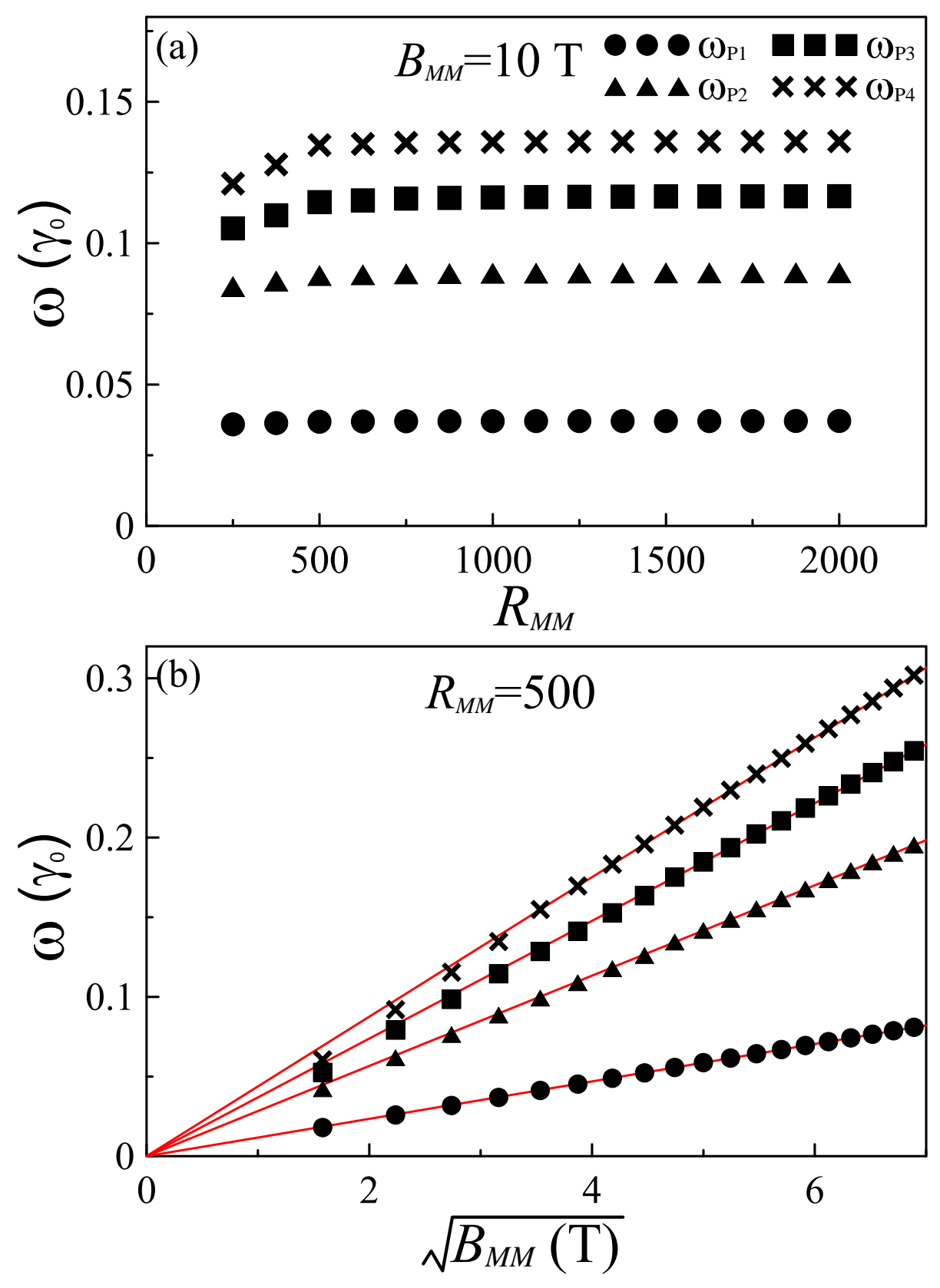

Figure 10.7. The dependence of the absorption frequency on (a) the period $R_{M M}$ and (b) the square root of field strength $B_{M M}$. 


\section{Spatially Modulated Electric Potential}

\subsection{Oscillation Energy Subbands}

Besides the spatially modulated magnetic field, the low-energy physical properties can also be strongly tuned by a spatially modulated electric potential. The energy bands for $V_{M E}=$ $0.05 \gamma_{0}$ and $R_{M E}=500$ are shown in Fig. 10.8. The unoccupied conduction subbands are symmetric to the occupied valence subbands about $E_{F}$. The parabolic subbands are nondegenerate and oscillate near $k_{y}=2 / 3$. There exists on intersection where two parabolic subbands cross each other at $E_{F}$. Each subband has several band-edge states, which lead to the prominent peaks in the DOS and optical absorption spectra. For convenience, these band-edge states are further divided into two categories called $\mu$ and $\nu$ states, as indicated in Fig. 10.8. The two $\mu(\nu)$ states at the left- and right-hand sites of $k_{y}=2 / 3$ might have a small difference in energies; that is, parabolic bands might be bilaterally asymmetric about $k_{y}=2 / 3$. Not far away from $k_{y}=2 / 3$, the energy subbands with linear dispersions intersect at $E_{F}$, preserving more Fermi-momentum states and forming several Dirac cones. Moreover, the number of Fermi-momentum states or Dirac cones increases with the potential strength and modulation period.

The optical absorption spectrum for $R_{M E}=500$ and $V_{M E}=0.05 \gamma_{0}$ along the armchair direction, as shown in Fig. 10.9 by the black solid curve, exhibits two groups of prominent peaks, $\Sigma_{n}$ 's and $\Upsilon_{n}$ 's. They are mainly due to the optical excitations from $\mu_{n}^{v}$ to $\mu_{n+1}^{c}$ $\left(\mu_{n+1}^{v}\right.$ to $\left.\mu_{n}^{c}\right)$ and $\mu_{n}^{v}$ to $\mu_{n+2}^{c}\left(\mu_{n+2}^{v}\right.$ to $\left.\mu_{n}^{c}\right)$, respectively. Moreover, with regard to the peak intensity, the peaks $\Sigma_{n}$ 's $\left(\Upsilon_{n}\right.$ 's) can be further divided into two subgroups. For example, the peak heights of $\Sigma_{1}, \Sigma_{3} ; \Sigma_{5}$, respectively, resulting from the transitions of $\mu_{1}^{v}$ to $\mu_{2}^{c}\left(\mu_{2}^{v}\right.$ to $\left.\mu_{1}^{c}\right), \mu_{3}^{v}$ to $\mu_{4}^{c}\left(\mu_{4}^{v}\right.$ to $\left.\mu_{3}^{c}\right) ; \mu_{5}^{v}$ to $\mu_{6}^{c}\left(\mu_{6}^{v}\right.$ to $\left.\mu_{5}^{c}\right)$ are very low, while the peaks $\Sigma_{2}, \Sigma_{4} ; \Sigma_{6}$ originating from the excitations $\mu_{2}^{v}$ to $\mu_{3}^{c}\left(\mu_{3}^{v}\right.$ to $\left.\mu_{2}^{c}\right), \mu_{4}^{v}$ to $\mu_{5}^{c}\left(\mu_{5}^{v}\right.$ to $\left.\mu_{4}^{c}\right) ; \mu_{6}^{v}$ to $\mu_{7}^{c}$ ( $\mu_{7}^{v}$ to

$\left.\mu_{6}^{c}\right)$ present much stronger intensities than the peaks $\Sigma_{1}, \Sigma_{3}$ and $\Sigma_{5}$. That is to say, the peak of $\Sigma_{2 n}$ 's are higher than those of $\Sigma_{2 n-1}$ 's in the group $\Sigma_{n}$. The peaks of $\Upsilon_{n}$ 's exhibit 


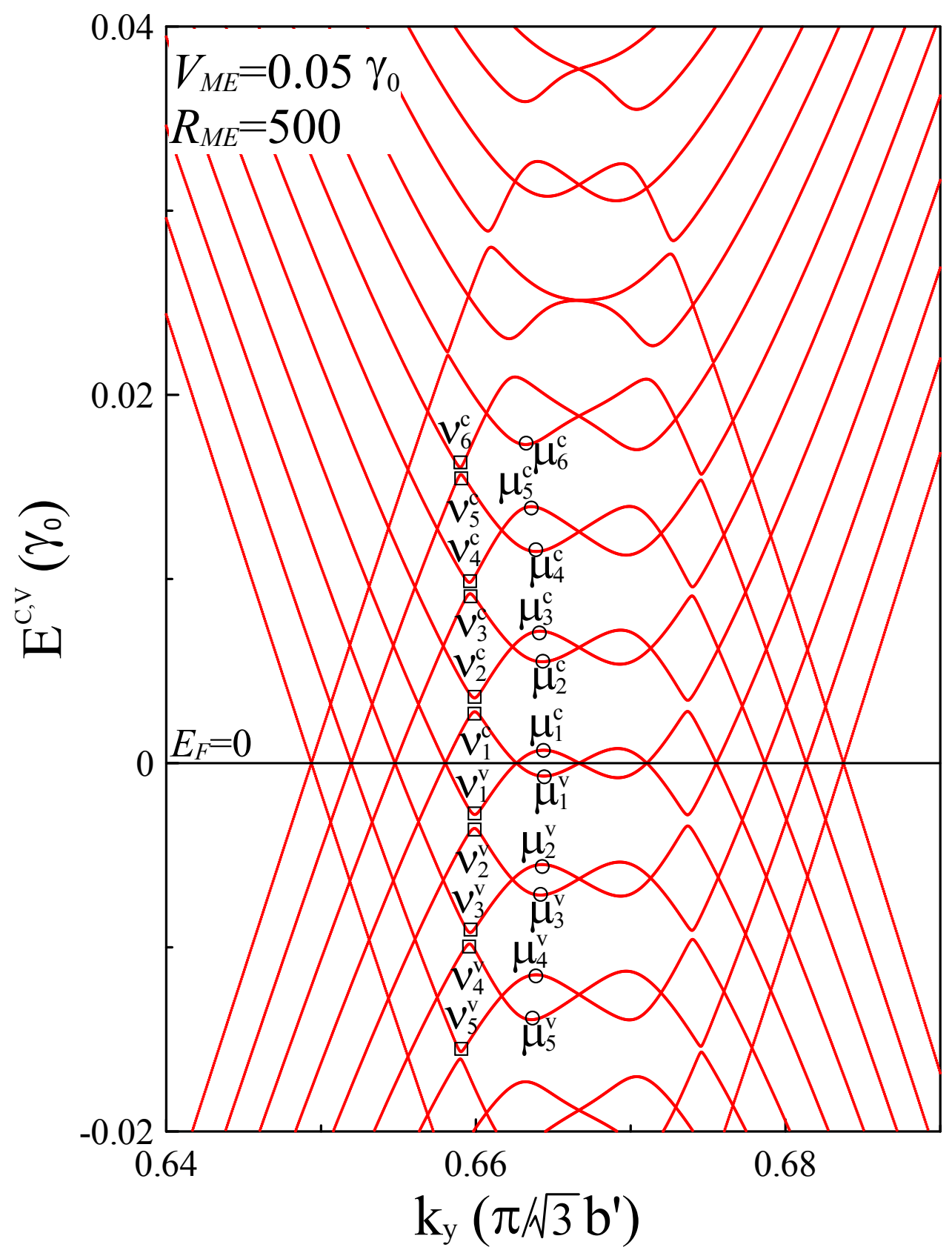

Figure 10.8. The energy dispersions for the modulated electric potential along the armchair direction with $R_{M M}=500$ and $V_{M E}=0.05 \gamma_{0}$. 
similar features to those of $\Sigma_{n}$ 's. For instance, peaks $\Upsilon_{1}, \Upsilon_{3} ; \Upsilon_{5}$, respectively arising from the transitions of $\mu_{1}^{v}$ to $\mu_{3}^{c}\left(\mu_{3}^{v}\right.$ to $\left.\mu_{1}^{c}\right), \mu_{3}^{v}$ to $\mu_{5}^{c}\left(\mu_{5}^{v}\right.$ to $\left.\mu_{3}^{c}\right) ; \mu_{5}^{v}$ to $\mu_{7}^{c}\left(\mu_{7}^{v}\right.$ to $\left.\mu_{5}^{c}\right)$, own the peaks with very weak intensities. In contrast to $\Upsilon_{1}, \Upsilon_{3} ; \Upsilon_{5}$, the peak intensities of $\Upsilon_{2}$ and $\Upsilon_{4}$ resulting from the excitations $\mu_{2}^{v}$ to $\mu_{4}^{c}\left(\mu_{4}^{v}\right.$ to $\left.\mu_{2}^{c}\right)$ and $\mu_{4}^{v}$ to $\mu_{6}^{c}\left(\mu_{6}^{v}\right.$ to $\left.\mu_{4}^{c}\right)$ are relatively stronger. Furthermore, the $\mu$ and $\nu$ states lead to different contributions to the two kinds of optical absorption peaks. Most peaks originating from the two different band-edge states have nearly the same frequencies, while the peak intensities are not the same. The blue and red curves correspond to the optical absorption spectra which contains only the excitations of $\mu$ and $\nu$ states, respectively. Except for the peak $\Sigma_{2}$ with comparable contributions which are attributed to the transitions of $\mu$ and $\nu$ states, the other peaks with different contributions from the two states have nearly the same frequency. The peaks from the $\mu$ states exhibit much stronger intensities than those from the $\nu$ states. In other words, peaks in the optical absorption spectrum mainly result from excitations of the $\mu$ states.

\subsection{Anisotropic Optical Absorption Spectra}

The polarization direction and the strength, period and direction of the modulating electric field strongly affect the features of the optical absorption spectrum. The spectra associated with $\widehat{\mathbf{E}} \perp \widehat{x}$ (black solid curve) and $\widehat{\mathbf{E}} \| \widehat{x}$ (red solid curve) for $R_{M E}=500$ and $V_{M E}=0.05$ $\gamma_{0}$ along the armchair direction and $R_{M E}=866$ and $V_{M E}=0.05 \gamma_{0}$ along the zigzag direction (blue solid curve) are shown in Fig. 10.10(a) for a comparison. Compared with

the results of $\widehat{\mathbf{E}} \perp \widehat{x}$ and $\widehat{\mathbf{E}} \| \widehat{x}$, the peak structures related to the two polarization directions are totally different, which reflect the anisotropic behavior of the polarization direction. Similarly, the anisotropy of the modulation directions is reflected by that the absorption spectra corresponding to the armchair and zigzag directions display distinct features, i.e., the anisotropic behavior of the polarization directions are more obvious than that in the MM case. With increasing the modulation strength to $V_{M E}=0.1 \gamma_{0}$ (red solid 


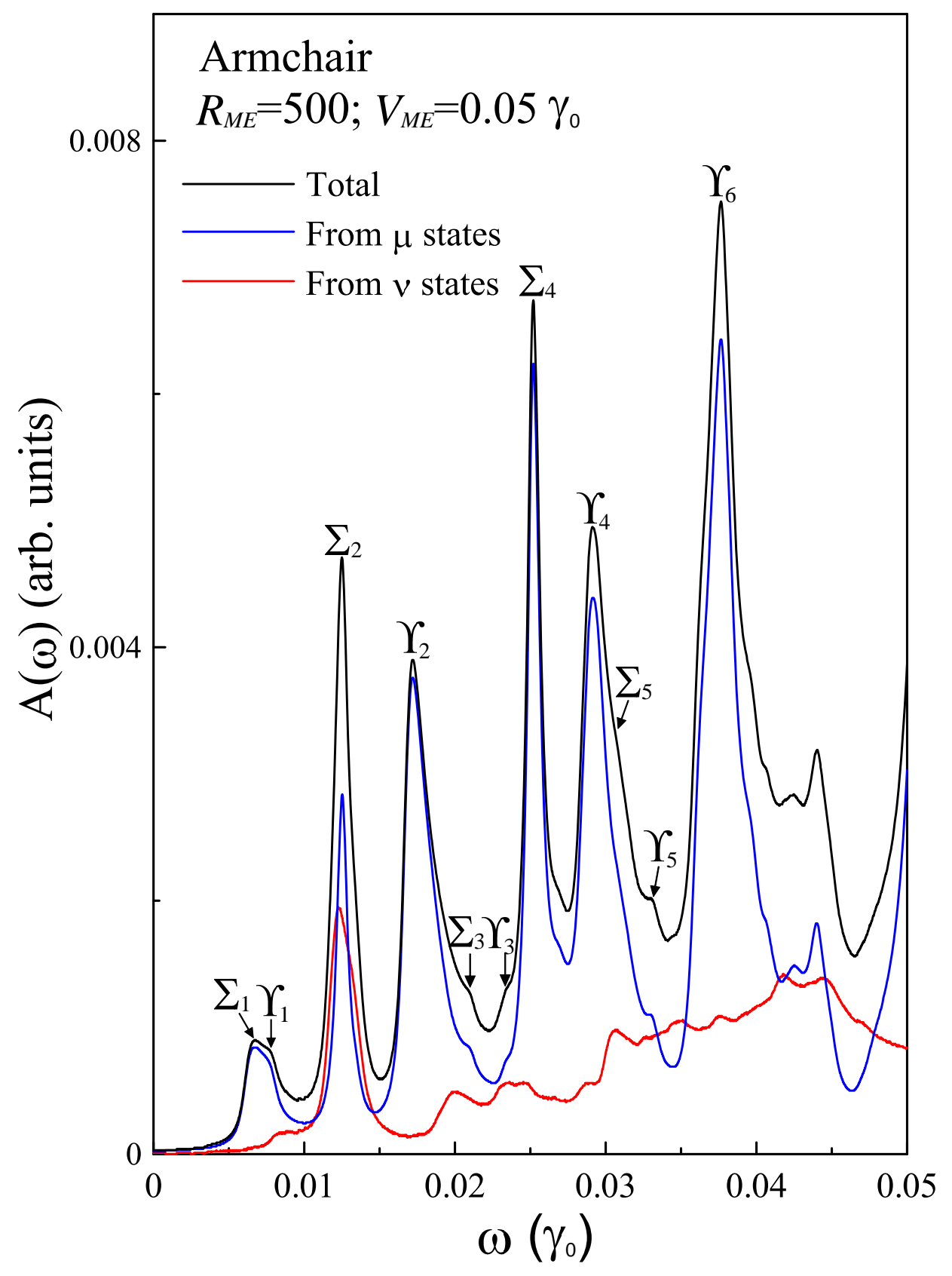

Figure 10.9. The optical absorption spectra corresponding to Fig. 10.8, which includes the contributions from the $\mu$ and $\nu$ states, respectively. 
curve in Fig. 10.10(b)), the results show that the peak intensity strongly depends on $V_{M E}$, but their relationship is not straight forward. For the modulation period, the spectra at a larger $R_{M E}=1000$ (blue solid curve) along the armchair direction present features diverse to those in the spectra at $R_{M E}=1000$. The peak number grows and the peak intensities decay with a increase of the period. A redshift occurs in longer periods. For example, the peak frequencies $\Sigma_{1}, \Sigma_{3} ; \Sigma_{5}$, as indicated in black and green curves, are almost reduced to half of the original ones when the modulation period is enlarged from 500 to 1000 .

The optical absorption spectra in the ME case do not reveal certain selection rules. This is due to the fact that the amplitudes $A_{\mathbf{o}}^{c, v}$ and $B_{\mathbf{o}}^{v, c}$ of the wave functions do not exist a simple relationship similar to that in the UM and MM cases. The wave functions in the modulated electric potential are no longer distributed around the center location; rather, they display standing-wave-like features in the primitive unit cell and are distributed over the entire primitive cell, as shown in Fig. 10.11. However, the wave functions of the edge-states $\mu$ and $\nu$ exhibit irregular behavior such as disordered numbers of zero points, asymmetric spatial distributions, and random oscillations. These irregular waveforms might result from different site energies for the carbon atoms in the modulated electric potential. 


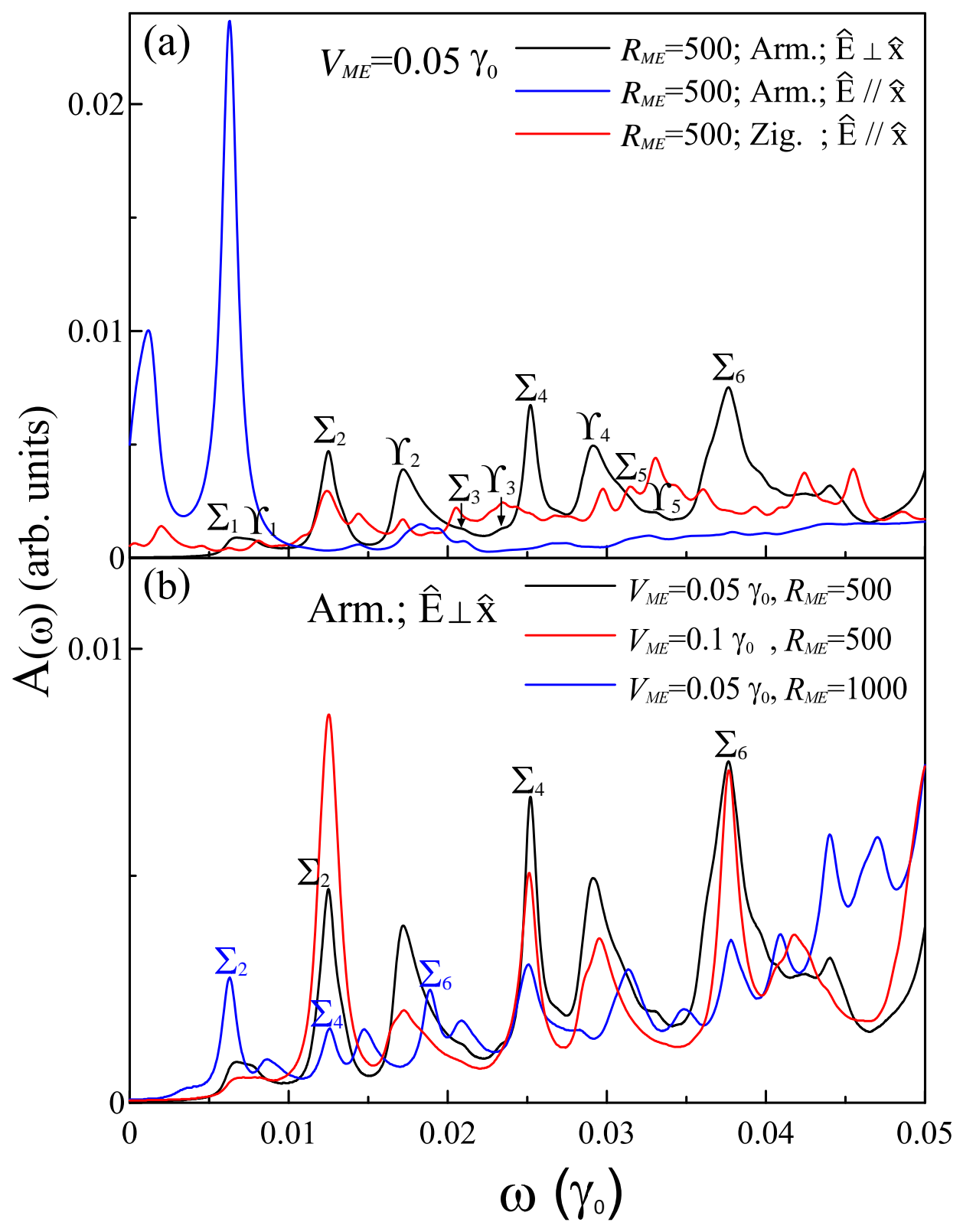

Figure 10.10. The optical absorption for (a) $V_{M E}=0.05 \gamma_{0}$ at a fixed periodic length with modulation and polarization along the armchair and zigzag directions and (b) different modulation periods and field strengths with both the modulation and polarization along the armchair direction. 


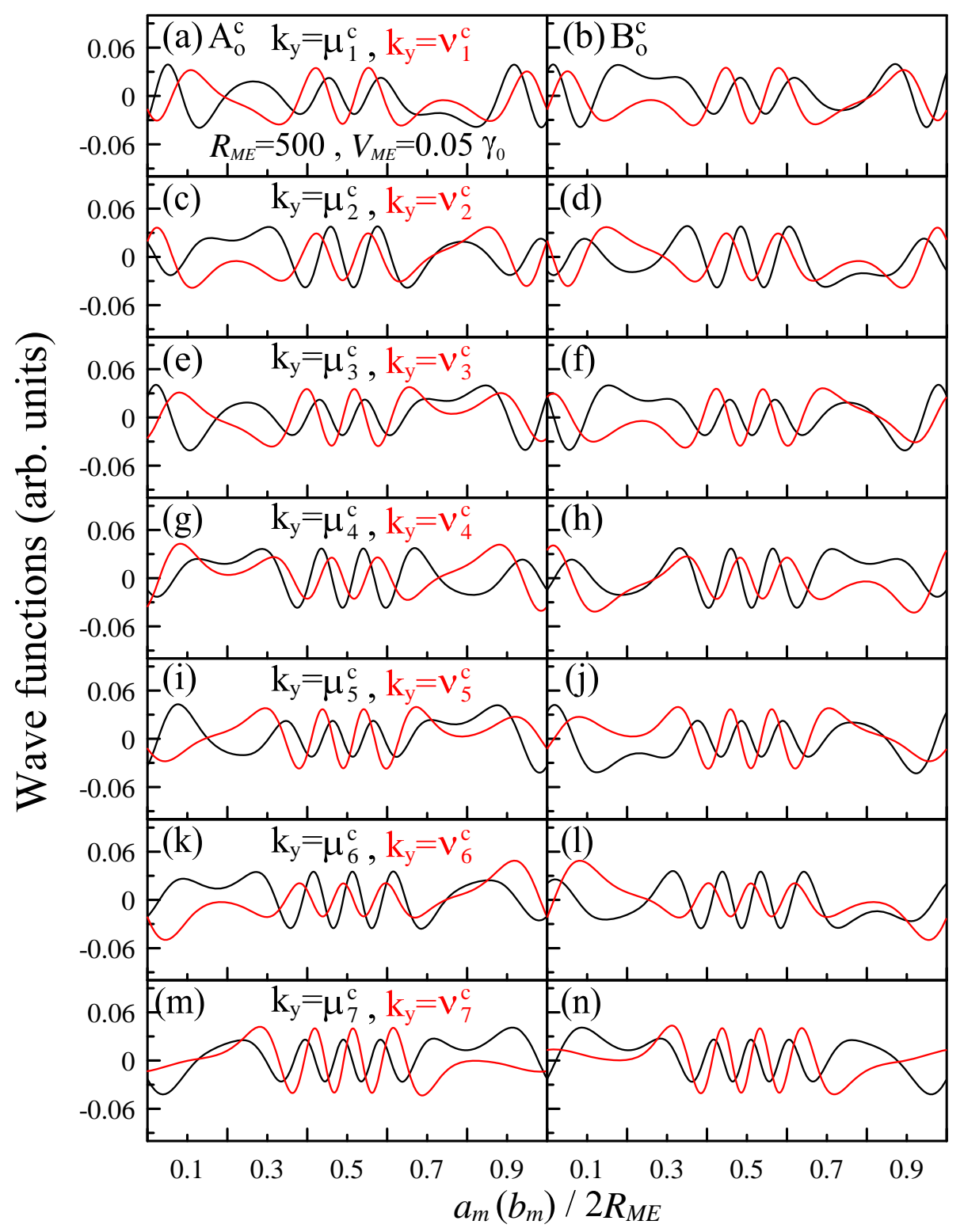

Figure 10.11. The wave functions at different band-edge states, the $\mu_{i}^{c}$ and $\nu_{i}^{c}$ states for $i=1^{\sim} 7$. 


\section{Uniform Magnetic Field Combined with Modulated Magnetic Field}

\subsection{Landau Level Spectra Broken by Modulated Magnetic Fields}

A further discussion of graphene in a composite field, the UM-MM case, is presented in this section. The main characteristics of the LLs at $B_{U M}=5 \mathrm{~T}$ are affected by the modulated magnetic field $\left(B_{M M}=1 \mathrm{~T}\right.$ and $\left.R_{M M}=395\right)$, as shown in Fig. 10.12 (a) by the black curves. The LL with $n^{c, v}=0$ at $E_{F}=0$ remains the same features of the UM case. On the other hand, each dispersionless LL with $n^{c, v} \geqslant 1$ splits into two parabolic subbands with double degeneracy. The subbands possess two kinds of band-edge states, $n^{c, v} \zeta$ and $n^{c, v} \eta$, which correspond to the minimum field strength $B_{U M}-B_{M M}$ and maximum field strength $B_{U M}+B_{M M}$, respectively. The surrounding electronic states at $n^{c, v} \eta$ congregate more easily, which results in the smaller band curvature. Comparably fewer states congregate at $n^{c, v} \zeta$, and the resulting band curvature is larger. Increasing $B_{M M}$ induces more complex energy spectra, as shown in Fig. 10.12 (b) for $R_{M M}=395$ and $B_{M M}=5 \mathrm{~T}$. The parabolic subbands with $n^{c, v} \geqslant 1$ display wider oscillation amplitudes, stronger energy dispersions, and greater band curvatures. The largest and smallest band curvatures occur at the local minima $n^{c, v} \zeta$ and local maxima $n^{c, v} \eta$ states, respectively. The subband amplitudes are nearly linearly magnified by $B_{M M}$ as $B_{M M} \leq B_{U M}$. It is noticeable that neither the minima of the conduction bands nor the maxima of the valence bands exceed $E_{F}=0$ even for $B_{M M}$ much larger than $B_{U M}$, as shown in Fig. $10.12(\mathrm{c})$ for $R_{M M}=395$ and $B_{M M}=40$ T. Thus no overlap exists between the conduction and valence bands, regardless of the modulation strength. With further increasing modulated field strength as $B_{M M} \gg B_{U M}$, the electronic structures are expected to approach to those in the MM case. 


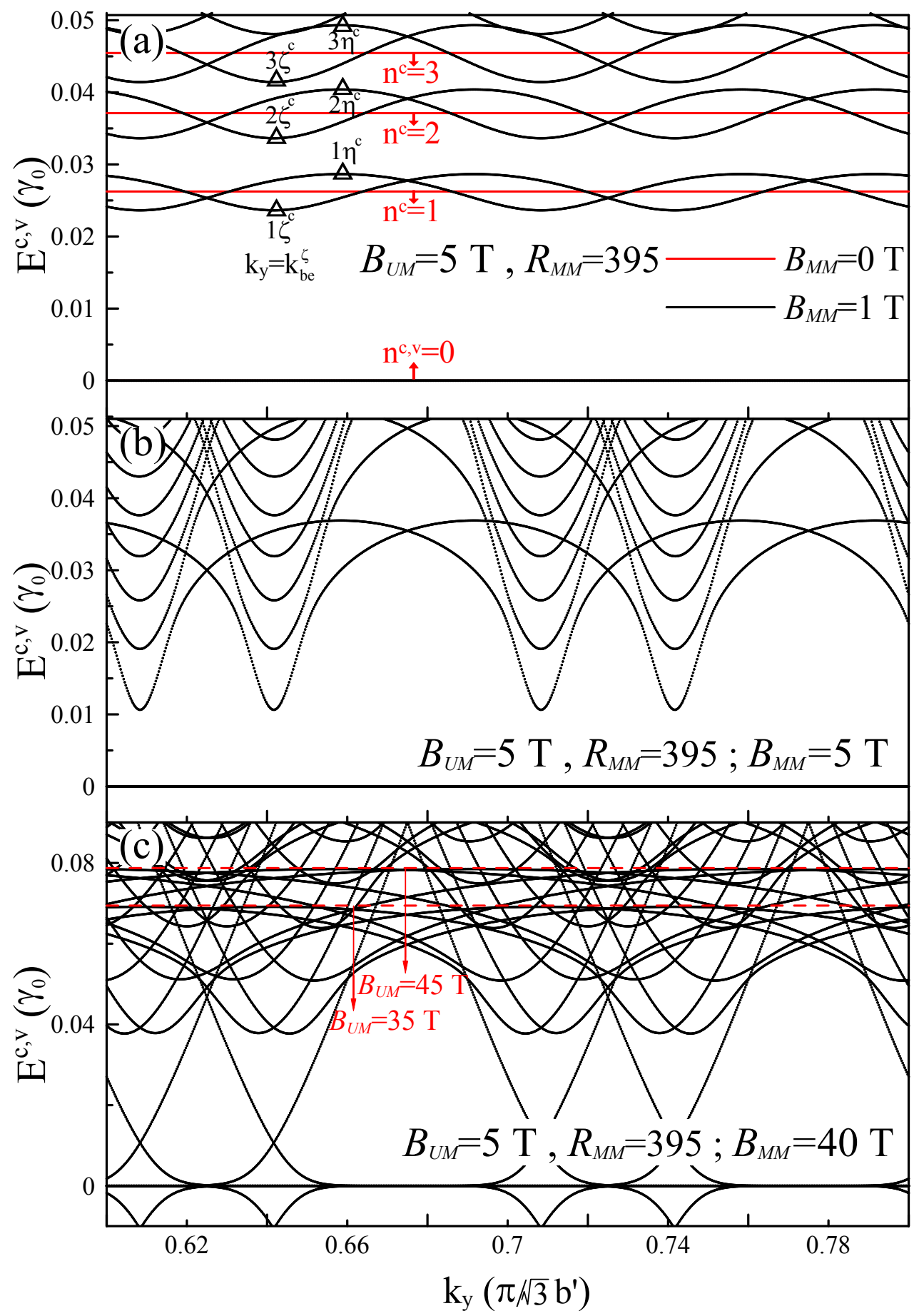

Figure 10.12. The energy dispersions for (a) the uniform magnetic field $B_{U M}=5 \mathrm{~T}$ by the red curves and the composite field $B_{U M}=5 \mathrm{~T}$ combined with $R_{M M}=395$ and $B_{M M}=1$ $\mathrm{T}$ by the black curves, (b) $B_{U M}=5 \mathrm{~T}$ combined with $R_{M M}=395$ and $B_{M M}=5 \mathrm{~T}$, and (c) $B_{U M}=5 \mathrm{~T}$ combined with $R_{M M}=395$ and $B_{M M}=40 \mathrm{~T}$. All modulated fields are applied along the armchair direction. 


\subsection{Symmetry Broken of Landau Level Wave Functions}

The LL wave functions modified by the modulated magnetic field are shown in Fig. 10.13. The spatial distributions corresponding to $k_{b e}^{\zeta}$, labeled in Fig. 10.12, exhibit slightly broadened and reduced amplitudes, as indicated by the black curves in Figs. 10.13(a)-(d) for $B_{M M}=1 \mathrm{~T}$. However, the spatial symmetry and the location centers of the wave functions remain unchanged. Under the influence of a small $B_{M M}$, the simple relation between $A_{\mathbf{o}}^{c, v}$ and $B_{\mathbf{o}}^{c, v}$ of the wave functions is almost preserved. However, a stronger modulation strength results in greater spatial changes of the wave functions, as shown in Figs. 10.13(e)-(f) for $B_{U M}=B_{M M}=5 \mathrm{~T}$. The increased broadening and asymmetry of the spatial distributions of the wave functions at $n^{c, v}=0$ are revealed. However, the spatial distributions with $n^{c, v} \geqslant 1$ are only widened (i.e., $n^{c}=1$ in Figs. $10.12(\mathrm{~g})$ and $(\mathrm{h})$ ), but the spatial symmetry is retained. With increasing $B_{M M}$, as shown in Figs 10.13(i)-(l) for $B_{M M}=40 \mathrm{~T}$, the symmetry of the wave functions with $n^{c, v}=0$ is recovered and one can expect that the main features of the wave functions will become similar to those in the MM case. Obviously, the electronic properties show critical changes as $B_{M M}$ equals $B_{U M}$, which should be reflected to the optical properties.

\subsection{Magneto-Optical Absorption Spectra with Extra Selection Rules}

The optical absorption spectra corresponding to Fig. 10.13 are shown in Figs. 10.14 and 10.15. In Fig. 10.14(a), the absorption spectra corresponding to the UM-MM case at $B_{U M}=5 \mathrm{~T}$ with $R_{M M}=395$ and $B_{M M}=1 \mathrm{~T}$ and the UM case at $B_{U M}=5 \mathrm{~T}$ are shown together for a comparison. The red curves coming from the LLs at $B_{U M}=5 \mathrm{~T}$ display delta-function-like peaks $\omega_{L L}^{n n^{\prime}}$ with the selection rule $\Delta n=1$. However, the modulated magnetic field modifies each delta-function-like peak into two split square-root-divergent

peaks, $\omega_{\zeta}^{n n^{\prime}}$ and $\omega_{\eta}^{n n^{\prime}}$, as shown by the black curves. Each $\omega_{\zeta}^{n n^{\prime}}\left(\omega_{\eta}^{n n^{\prime}}\right)$ originates from the 


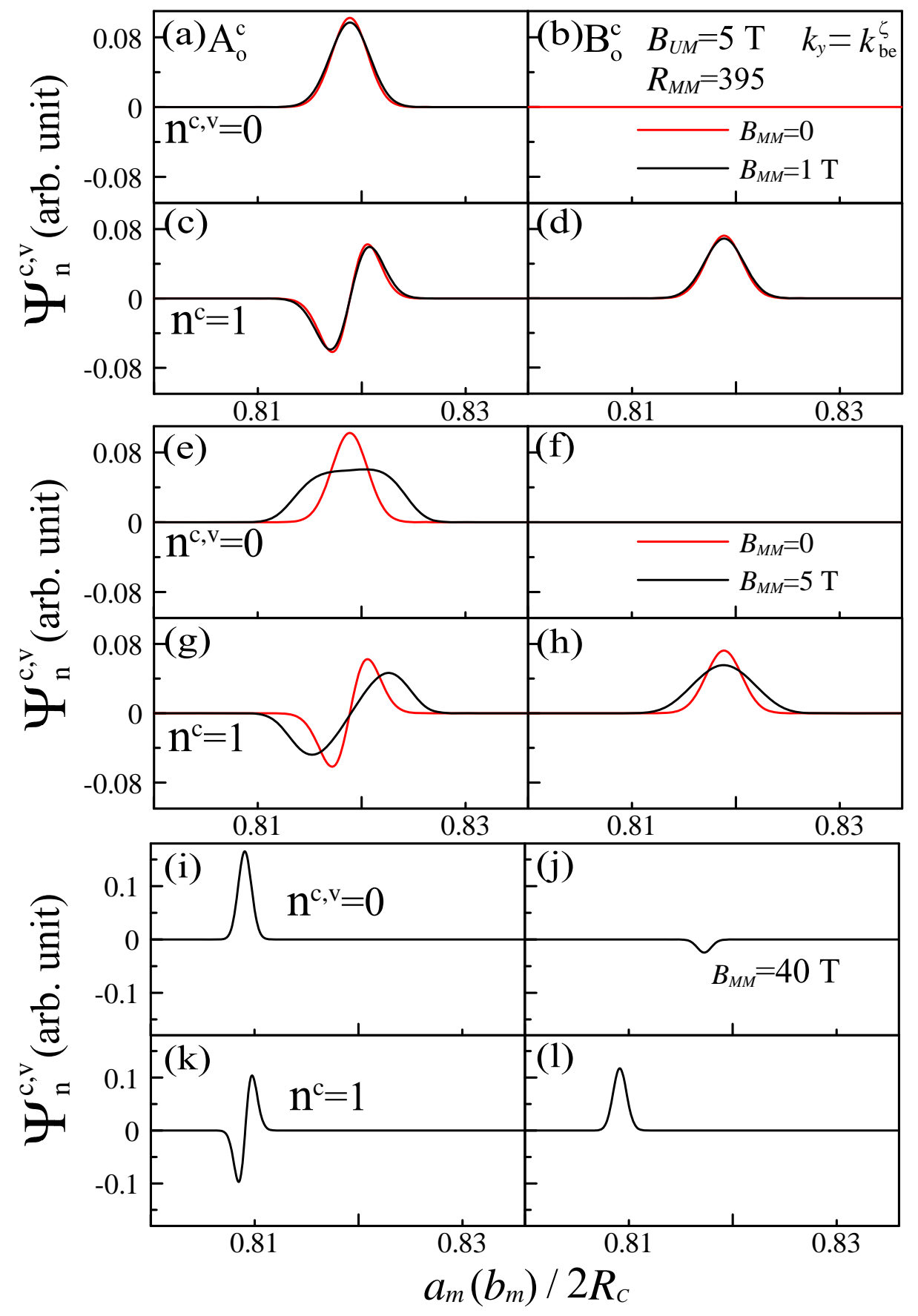

Figure 10.13. The wave functions with $n^{c, v}=0$ and $n^{c}=1$ at $k_{b e}^{\zeta}$ for (a)-(d) the uniform magnetic field $B_{U M}=5 \mathrm{~T}$ by the red curves and the composite field $B_{U M}=5 \mathrm{~T}$ combined with $R_{M M}=395$ and $B_{M M}=1 \mathrm{~T}$ by the black curves, (e)-(h) $B_{U M}=5 \mathrm{~T}$ by the red curves and $B_{U M}=5 \mathrm{~T}$ combined with $R_{M M}=395$ and $B_{M M}=5 \mathrm{~T}$ by the black curves, and (i)-(l) $B_{U M}=5 \mathrm{~T}$ combined with $R_{M M}=395$ and $B_{M M}=40 \mathrm{~T}$. 
transitions of $n \zeta \rightarrow n+1 \zeta$ and $n+1 \zeta \rightarrow n \zeta(n \eta \rightarrow n+1 \eta$ and $n+1 \eta \rightarrow n \eta)$ and its absorption frequency is same as that generated from the LLs at $B_{U M}-B_{M M}=4 \mathrm{~T}$ $\left(B_{U M}-B_{M M}=6 \mathrm{~T}\right)$. These absorption peaks obey a selection rule, $\Delta n=1$, similar to that in the UM case.

With increasing the modulated field strength as $B_{M M}=B_{U M}=5 \mathrm{~T}$, the absorption spectrum has evident variety, as shown in Fig. 10.14(b). In addition to the peaks $\omega_{\zeta}^{n n^{\prime}}$ and $\omega_{\eta}^{n n^{\prime}}$ with the selection rule $\Delta n=1$, two extra peaks with $\Delta n=2$ and $3, \omega_{\zeta}^{02}$ and $\omega_{\zeta}^{03}$, are generated. These two peaks do reflect the fact that the wave functions of the LLs with $n^{c, v}=0$ are destroyed by the modulated magnetic field. As the modulated field strength further raises to $B_{M M}=40 \mathrm{~T}$ (red dashed curves Fig. 10.15), the spectrum displays some features similar to those of the spectrum in the MM case at $R_{M M}=395$ and $B_{M M}=40 \mathrm{~T}$ (black solid curves in Fig. 10.15), i.e., the principal peaks $\omega_{P}$ 's and the subpeaks $\omega_{S}$ 's in the MM case are also shown in the UM-MM case as $B_{M M}>B_{U M}$. Moreover, the subpeaks features, which are associated with the positions at the net field strength equal to zero, are almost the same in both the MM and UM-MM case. The principal peaks, however, possess a pair structure with $\omega_{P n}^{-}$and $\omega_{P n}^{+}$, which respectively correspond to two different field strengths, $\left|B_{U M}-B_{M M}\right|=35 \mathrm{~T}$ and $\left|B_{U M}+B_{M M}\right|=45 \mathrm{~T}$, and thus the difference between two field strengths lead to distinct absorption frequencies. For $B_{M M} \gg B_{U M}$, one can anticipate that the frequency discrepancy between the pair $\omega_{P n}^{-}$and $\omega_{P n}^{+}$becomes very small and then they will merge into one single peak, $\omega_{P n}$, i.e., the absorption spectrum restores to that in the pure MM case.

The dependence of the absorption frequency on the modulated field strength is shown in Fig. 10.16 for $B_{M M} \leq 5 \mathrm{~T}$. In the range of $B_{M M} \leq B_{U M}$, each of absorption peaks $\omega_{\zeta}^{n n^{\prime}}$ and $\omega_{\eta}^{n n^{\prime}}$ is linearly dependent on $B_{M M}$. This reflects the fact that the subband amplitudes are nearly linearly magnified by $B_{M M}$ within the range. However, in the higher absorption frequency region or the field range of $B_{M M}>B_{U M}$, the linear-dependence relationship will be broken since the subbands become overlapping and the subband amplitudes are not 


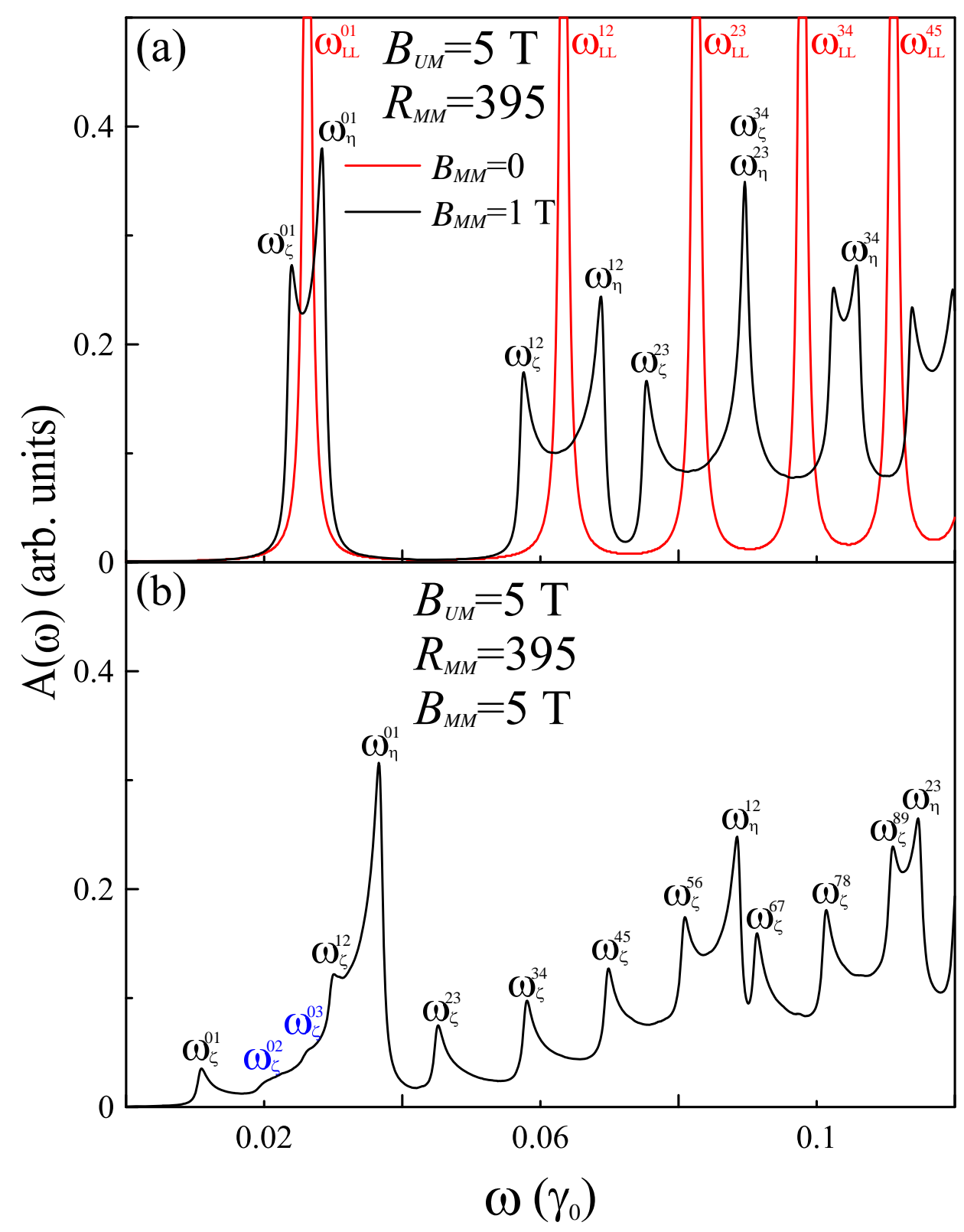

Figure 10.14. The optical absorption spectra corresponding to (a) the uniform magnetic field $B_{U M}=5 \mathrm{~T}$ by the red curve and the composite field $B_{U M}=5 \mathrm{~T}$ combined with $R_{M M}=395$ and $B_{M M}=1 \mathrm{~T}$ by the black curve and (b) the composite field $B_{U M}=5 \mathrm{~T}$ combined with $R_{M M}=395$ and $B_{M M}=5 \mathrm{~T}$. 


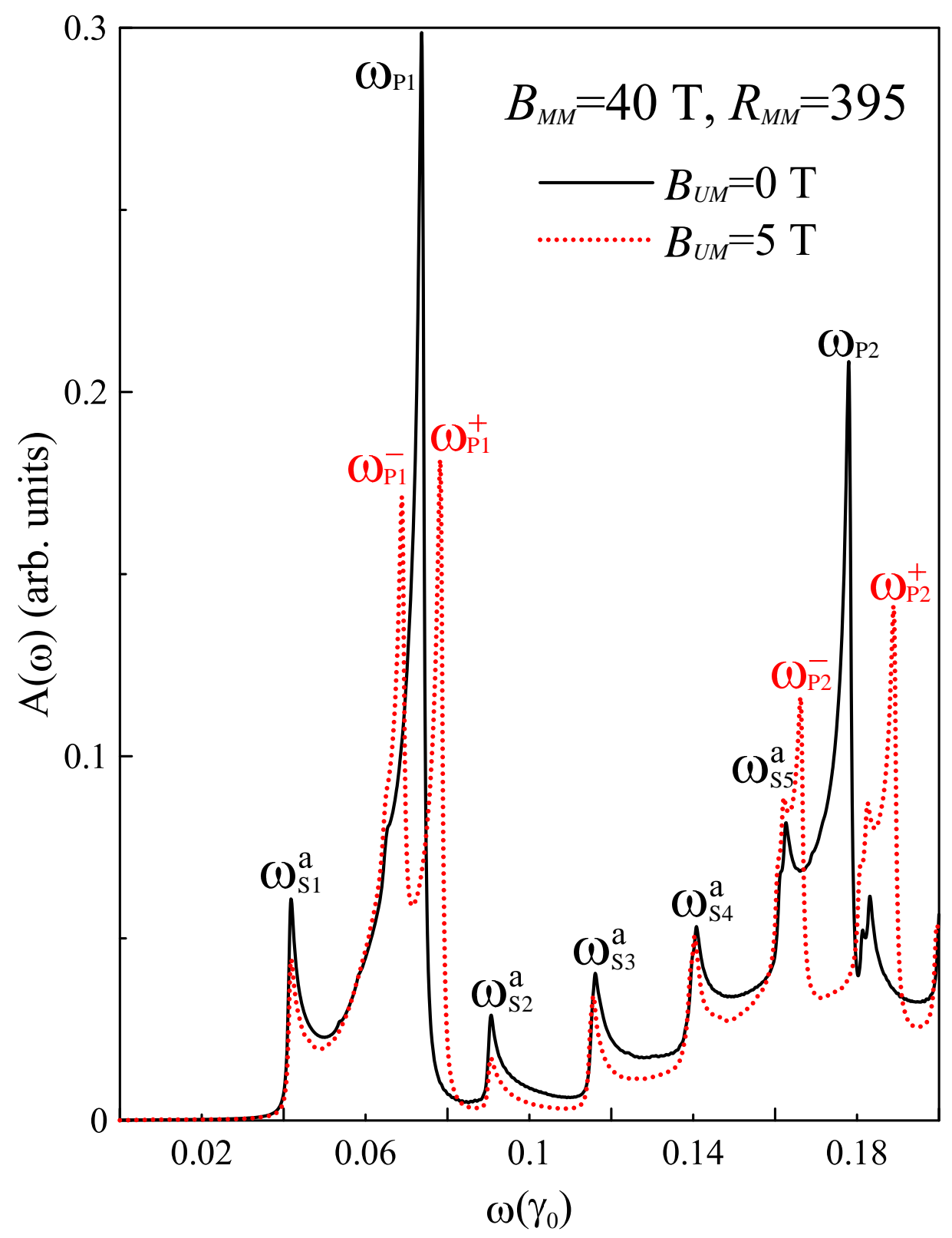

Figure 10.15. The optical absorption spectra corresponding to the composite field $B_{U M}=$ $5 \mathrm{~T}$ combined with $R_{M M}=395$ and $B_{M M}=40 \mathrm{~T}$ by the red dotted curve and the pure modulated magnetic field $R_{M M}=395$ and $B_{M M}=40 \mathrm{~T}$ by the black curve. 
linearly magnified by $B_{M M}$ anymore. 


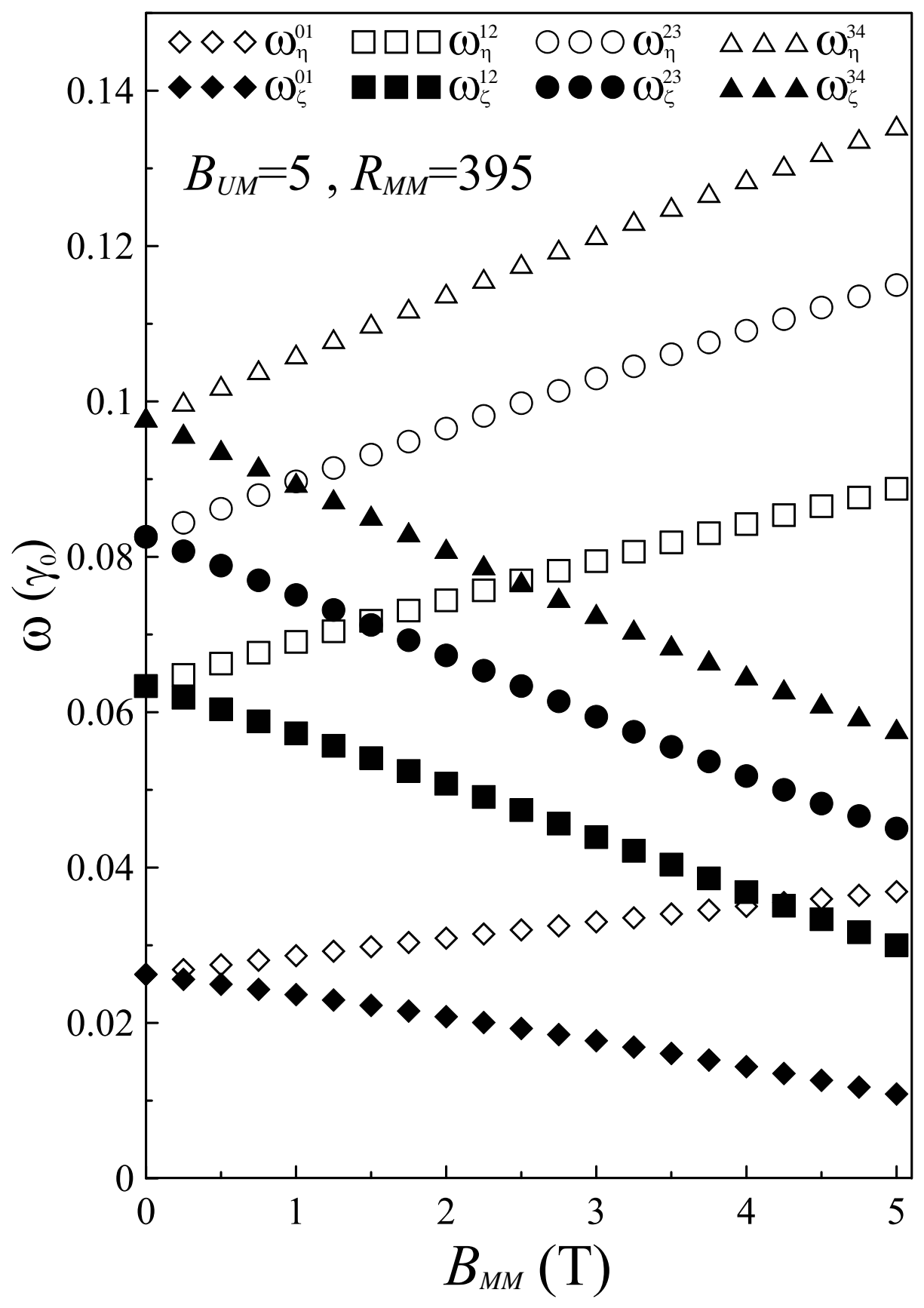

Figure 10.16. The dependence of absorption frequencies, $\omega_{\eta}^{n n^{\prime}}$ and $\omega_{\zeta}^{n n^{\prime}}$ with $|\Delta n|=$ $\left|n-n^{\prime}\right|=1$, on the modulated strength $B_{M M}$. 


\section{Uniform Magnetic Field Combined with Modulated Electric Potential}

\subsection{Landau Level Spectra Broken by Modulated Electric Poten- tials}

Compared with the situation in a modulated magnetic field, a modulated electric potential creates distinct effects on the LLs, as shown in Fig. 10.17(a) by the black curves for . The 0D LLs become the 1D sinusoidal energy subbands when electronic states are affected by the periodic electric potential. Each LL with four-fold degeneracy is split into two doubly degenerate Landau subbands (LSs), as shown in Fig. 10.17(a) by the black curves. Each LS owns two types of extra band-edge states, $k_{b e}^{\varkappa}$ and $k_{b e}^{o}$. For the conduction (valence) LSs, the band-edge states of $k_{b e}^{\varkappa}$ and $k_{b e}^{o}\left(k_{b e}^{e}\right.$ and $\left.k_{b e}^{\varkappa}\right)$ are, respectively, related to the wave functions, which possess a localization center at the minimum and maximum electric potentials (discussed in the ME case). It should be noted that the two LSs of $n^{c, v}=0$ only have the $\varrho$-type band-edge states. Under a small modulation strength, the energy spacings $\left(E_{s}\right.$ 's) or band curvatures for both $k_{b e}^{\varkappa}$ and $k_{b e}^{o}$ are almost the same, where $E_{s}$ is the spacing between a LS and a LL at $k_{b e}^{\varkappa}$ or $k_{b e}^{o}$. On the other hand, when the modulation strength is sufficiently large (e.g., $V_{M E}=0.02 \gamma_{0}$ in Fig. 10.17(b)), the energy dispersions of LSs are relatively strong and $E_{s}$ decreases with increasing state energies, i.e., the oscillations of LSs decline with an increase of $n^{c, v}$. In comparison to the $k_{b e}^{\varrho}$ state, the $k_{b e}^{\varkappa}$ state owns the smaller energy spacing and band curvature. Such differences are associated with the localization of the wave function within the potential well. 


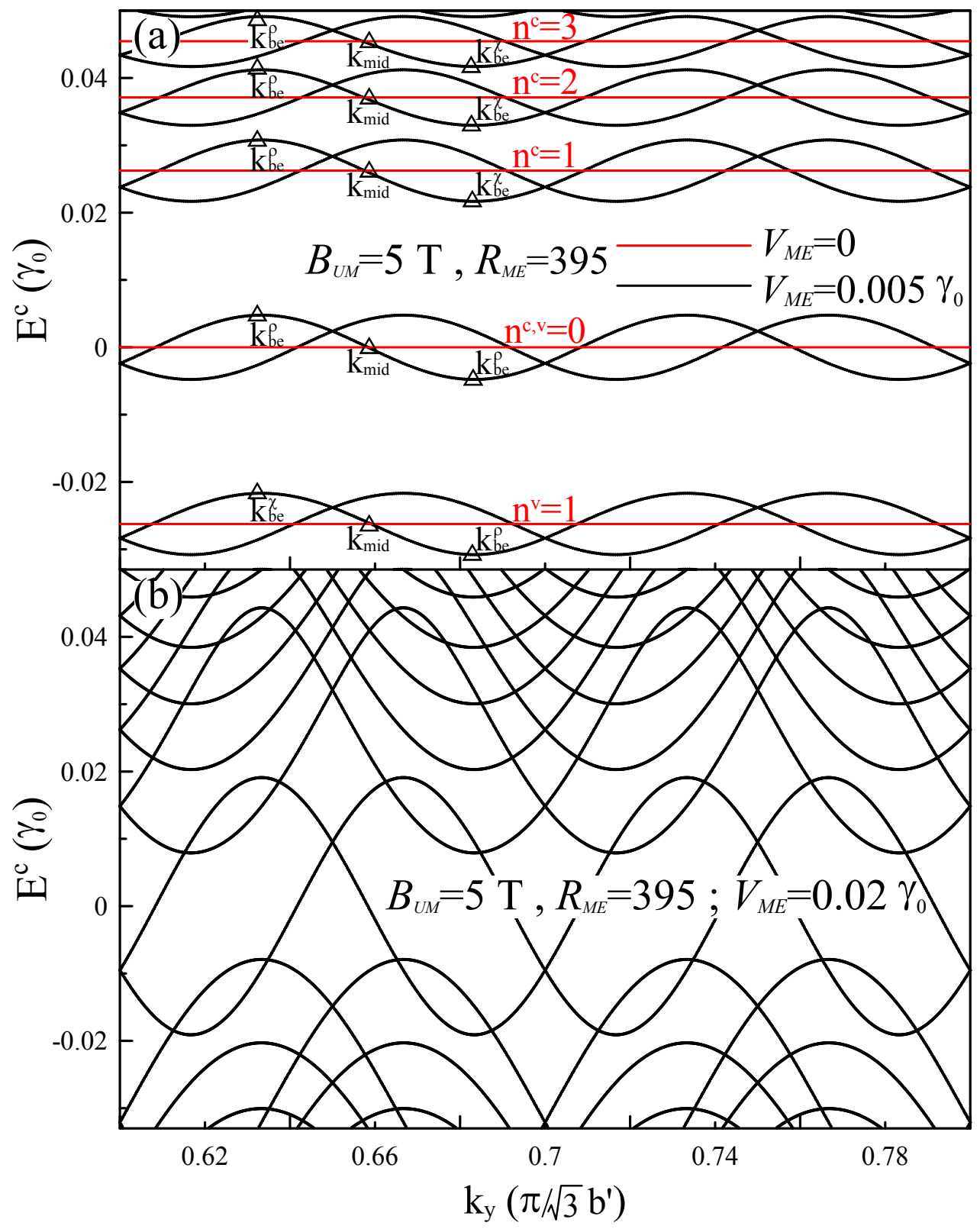

Figure 10.17. The energy dispersions for (a) the uniform magnetic field $B_{U M}=5 \mathrm{~T}$ by the red curves and the composite field $B_{U M}=5 \mathrm{~T}$ combined with $R_{M M}=395$ and $V_{M E}=0.005 \gamma_{0}$ by the black curves, (b) $B_{U M}=5 \mathrm{~T}$ combined with $R_{M M}=395$ and $V_{M E}=0.02 \gamma_{0}$. All modulated fields are applied along the armchair direction. 


\subsection{Landau Level Wave Functions Broken by Modulated Electric Potentials}

The main features of the LL wave functions are altered by the modulated electric potential. The illustrated wave functions at the band-edge states and the midpoint $\left(k_{m i d}\right.$, indicated in Fig. 10.17(a)) between two band-edge states are used to examine the modulation effects. The wave functions at $k_{m i d}$ are modified by $V_{M E}(x)$, as shown in Figs. 10.18(a)-(h) for $B_{U M}=5 \mathrm{~T}$ and $R_{M E}=395$ at $V_{M E}=0,0.005$ and $0.02 \gamma_{0} . A_{\mathrm{o}}^{c, v}$ of $n^{c, v}=0$ is slightly reduced, while $B_{\mathbf{o}}^{c, v}$ of $n^{c, v}=0$ is slightly increased (Figs. 10.18(a) and 10.18(b)) after $V_{M E}$ is introduced. This means that carriers are transferred between the $a$ - and $b$-sublattices. With an increasing $n^{c, v}$, the spatial distribution symmetry of the LL wave functions is broken. The conduction and valence wave functions are, respectively, shifted toward the $+\widehat{x}$ and $-\widehat{x}$ directions, as shown in Figs. 10.18(e)-(h) for example. The proportionality relationship between $A_{\mathbf{o}}^{c, v}$ of $n^{c, v}$ and $B_{\mathbf{o}}^{c, v}$ of $n^{c, v}+1$ no longer exists, and neither do the relationships $A_{\mathbf{o}}^{c}=A_{\mathbf{o}}^{v}$ and $B_{\mathbf{o}}^{c}=-B_{\mathbf{o}}^{v}$. Moreover, the stronger $V_{M E}$ leads to greater changes in the spatial distributions of the wave functions. The spatial distributions of the wave functions strongly depend on $k_{y}$. As for the band-edge states, the aforementioned relationships of the wave functions are absent when $n^{c, v}$ 's are sufficiently large enough. For small $n^{c, v}$ 's (Figs. 10.18(i)-(l)), wave functions are less influenced by the modulated electric potential. However, the spatial distribution of LS with a larger $n^{c, v}$ becomes wider (narrower) for the $k_{b e}^{\varrho}\left(k_{b e}^{\varkappa}\right)$ state, as shown in Figs. 10.18(m)-(p). Moreover, the localization center of the band-edge states is hardly affected by $V_{M E}(x)$.

\subsection{Magneto-Optical Absorption Spectra Destroyed by Modu- lated Electric Potentials}

Under a modulated electric potential, the changes in the electronic properties of LLs are manifested in the optical absorption spectra. Each LL is split into two kinds of sinusoidal 

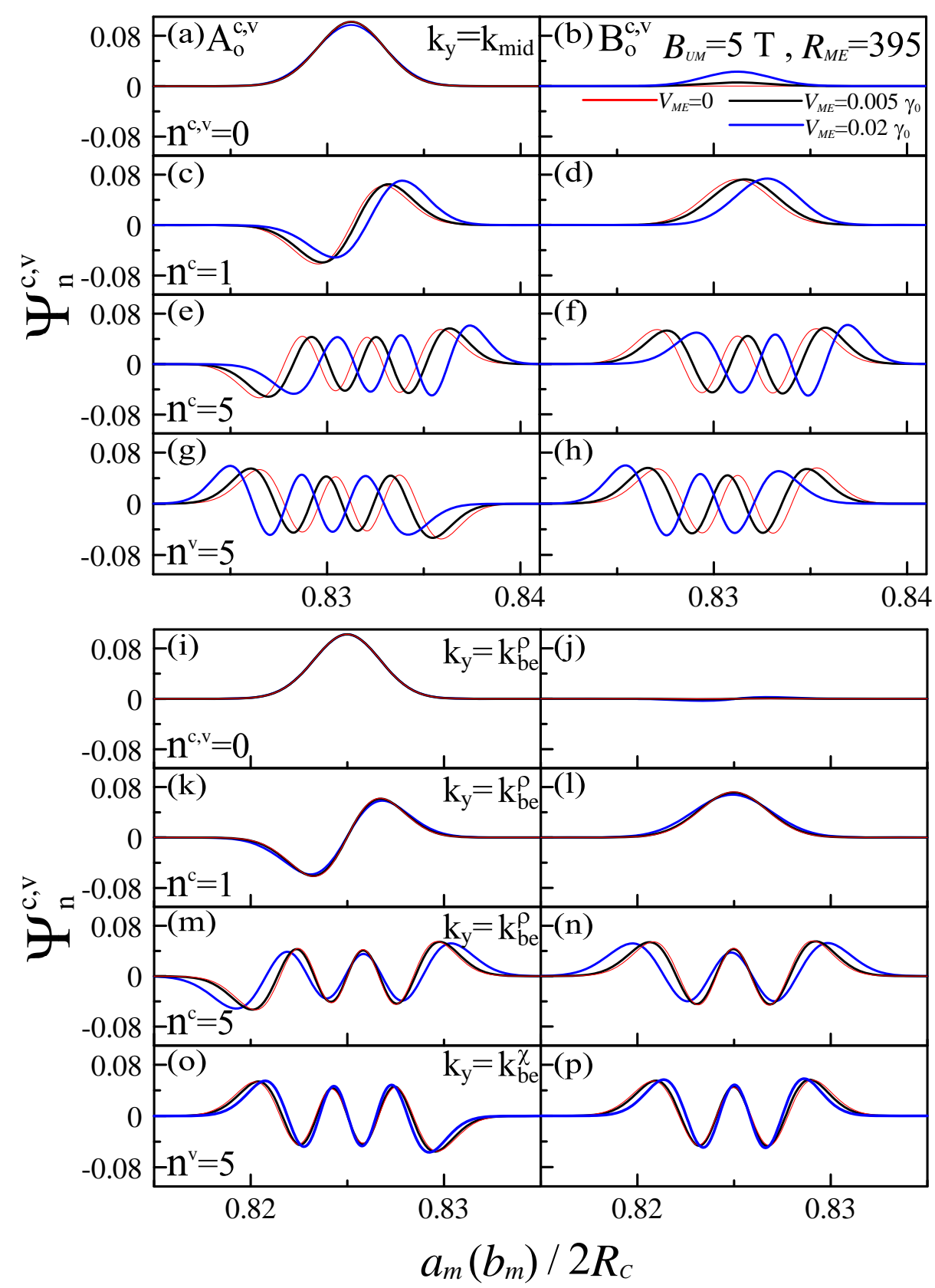

Figure 10.18. The wave functions for (a)-(d) $n^{c, v}=0, n^{c}=1, n^{c}=5$, and $n^{v}=5$ at $k_{\text {mid }}$, (i)-(n) $n^{c, v}=0, n^{c}=1$ and $n^{c}=5$ at $k_{b e}^{\rho}$, and (l)-(m) $n^{v}=5$ at $k_{b e}^{\chi}$. The results corresponding to the uniform magnetic field $B_{U M}=5 \mathrm{~T}$, the composite field $B_{U M}=5$ $\mathrm{T}$ combined with $R_{M M}=395$ and $V_{M E}=0.005 \gamma_{0}$, and $B_{U M}=5 \mathrm{~T}$ combined with $R_{M M}=395$ and $V_{M E}=0.02 \gamma_{0}$ are indicated by the red, black, and blue curves respectively. 
subbands, with one leading the other by $1 / 6$ of a period (Fig. 10.17(a)). The spatial localization region of the wave function is completely different between two kinds of subbands (not shown), but identical in corresponding to the same kind of LSs with different quantum numbers at the same $k_{y}$. This indicates that the optical transition between two different kinds of subbands is forbidden. The absorption spectrum for $R_{M E}=395$ at $V_{M E}=0.005 \gamma_{0}$ is shown in Fig. 10.19(a) by the black line. Each absorption peak, $\omega^{n n^{\prime}}$, originates from a transition between two LSs with quantum numbers $n$ and $n^{\prime}$ for the same kind of subbands. In addition to the original peaks, which correspond to the selection rule $\Delta n=1$ similar to that of LLs, there are extra peaks not characterized by the same selection rule. In the frequency range $\omega<0.1 \gamma_{0}$, the peak intensity of $\omega_{L L}^{n n+1}$, significantly reduced by $V_{M E}$, declines as the frequency increases. The extra peaks with $\Delta n \neq 1$ behave the opposite way. For a small $V_{M E}$, the peaks with $\Delta n=1$ are much stronger than those with $\Delta n \neq 1$.

The original and extra peaks can be explained by the subband transitions associated with a certain set of $k_{y}$ points. For the $k_{b e}^{\varkappa(\varrho)} \rightarrow k_{b e}^{\varrho(\varkappa)}$ transitions, the corresponding bandedge states have a high DOS and the symmetry of wave function is little changed. Therefore, they can promote the prominent peaks consistent with the $\Delta n=1$ selection rule. As shown in Fig. 10.19(b) by the thin dashed lines, $\omega_{\varkappa \varrho}^{n n+1}$ and $\omega_{\varrho \varkappa}^{n n+1}$ represent the absorption peaks from the transition channels $\left[n k_{b e}^{\varkappa} \rightarrow(n+1) k_{b e}^{\varrho},(n+1) k_{b e}^{\varrho} \rightarrow n k_{b e}^{\varkappa}\right]$ and $\left[n k_{b e}^{\varrho} \rightarrow(n+1) k_{b e}^{\varkappa}\right.$, $\left.(n+1) k_{b e}^{\varkappa} \rightarrow n k_{b e}^{\varrho}\right]$, respectively. These two peaks are close to each other, and almost overlap with the original peaks. But in cases where either $n$ or $n^{\prime}$ is zero, only the peak $\omega_{\varrho \varkappa}^{01}$ can be created by two transition channels: $\left[0 k_{b e}^{\varrho} \rightarrow 1 k_{b e}^{\varkappa}, 1 k_{b e}^{\varkappa} \rightarrow 0 k_{b e}^{\varrho}\right]$. The reduction of the transition channels is due to the fact that the $n^{c, v}=0$ subbands oscillate between the conduction and valence bands. For the $k_{m i d} \rightarrow k_{m i d}$ transitions, the significant change to the symmetry of the wave function results in different selection rules, i.e., $\Delta n \neq 1$. As shown in Fig. 10.19(b) by the thick dashed lines, the absorption peak $\omega_{m i d}^{n n^{\prime}}$ corresponds to the transitions between two LSs of $n^{v(c)}$ and $n^{\prime c(v)}$ from the $k_{\text {mid }}$ states. These types of peaks are responsible for the extra peaks in Fig. 10.19 (a), i.e., the $\Delta n \neq 1$ peaks primarily 


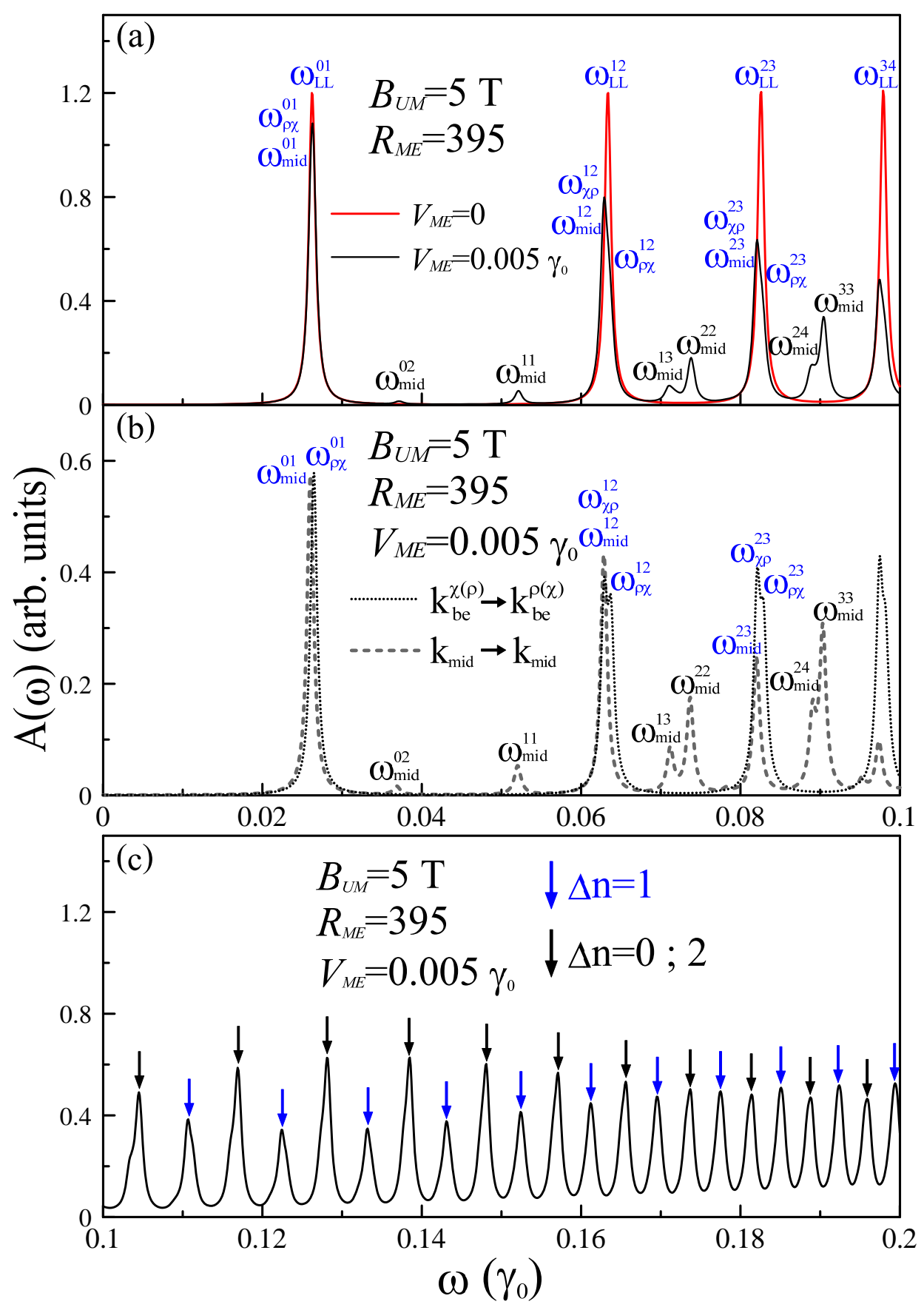

Figure 10.19. The optical absorption spectra corresponding to (a) the uniform magnetic field $B_{U M}=5 \mathrm{~T}$ by the red curve and the composite field $B_{U M}=5 \mathrm{~T}$ combined with $R_{M M}=395$ and $V_{M E}=0.005 \gamma_{0}$ by the black curve, (b) the transitions of $k_{b e}^{\chi(\rho)} \rightarrow k_{b e}^{\rho(\chi)}$ and $k_{\text {mid }} \rightarrow k_{\text {mid }}$ for $B_{U M}=5 \mathrm{~T}$ combined with $R_{M M}=395$ and $V_{M E}=0.005 \gamma_{0}$ by the dotted and dashed curves respectively, and (c) the higher frequency region of $0.1^{\sim} 0.2 \gamma_{0}$. 
arise from the middle states at lower modulation strength. The $k_{m i d} \rightarrow k_{m i d}$ transitions also contribute to the original peaks. Such contributions decline with an increased frequency. However, the $\Delta n=1$ absorption peaks are dominated by both the band-edge and middle states.

The peak intensities from different selection rules change dramatically with respect to the variation in frequency. The intensities of the $\Delta n=1$ peaks gradually rise for a further increase in frequency, while the opposite is true for those of the $\Delta n \neq 1$ peaks (Fig. 10.19(c)). Within the frequency range of $0.1 \gamma_{0}<\omega<0.2 \gamma_{0}$, the former is lower than the latter. The main reason for this is the fact that the symmetry violation of the wave functions is enhanced for LSs with larger $n^{c, v}$ s. It is deduced that the extra absorption peaks of $\Delta n \neq 1$ are relatively easily observed for experimental measurements at higher frequencies. Each of them is composed of two peaks, $\omega_{\text {mid }}^{n n}$ and $\omega_{\text {mid }}^{n-1 n+1}$, which satisfy $\Delta n=0$ and $\Delta n=2$ with nearly the same frequency.

The absorption spectrum exhibits more features for a higher modulated potential, as shown in Fig. 10.20 for $R_{M E}=395$ at $V_{M E}=0,0.02 \gamma_{0}$. In Fig. 10.20(a), the intensity of the extra peaks becomes comparable to that of the original peaks. Unlike in the lower $V_{M E}$ case (Fig. 10.19(a)), the peak intensities, regardless of their types, vary irregularly with the frequency. The oscillations of the subband structure are obviously augmented and cause the subband transitions to change greatly with respect to $k_{y}$. As a result, the absorption peaks grow much wider and split more evenly. Peaks generated by different selection rules are likely to appear, owing to the severe breakdown of the spatial symmetry of the wave functions. It should be noted that it is difficult to distinguish the original from the extra peaks solely based on the peak heights. Besides the aforementioned spectrum analysis, further discussions are made regarding two specific $k_{y}$ points. For the $k_{b e}^{\varkappa(\varrho)} \rightarrow k_{b e}^{\varrho(\varkappa)}$ transitions, $\varkappa$ - and $\varrho$-type band-edge states show different behavior in terms of energy spacing and curvature such that the two transition channels $\left[n k_{b e}^{\varkappa} \rightarrow(n+1) k_{b e}^{\varrho},(n+1) k_{b e}^{\varrho} \rightarrow\right.$ $\left.n k_{b e}^{\varkappa}\right]$ and $\left[n k_{b e}^{\varrho} \rightarrow(n+1) k_{b e}^{\varkappa},(n+1) k_{b e}^{\varkappa} \rightarrow n k_{b e}^{\varrho}\right]$ possess distinct frequencies (thin dashed 
line in Fig. 10.20(b)). Therefore, the initially coinciding peaks split up, and the original intensities are divided into fractions. Moreover, the band-edge states can induce the extra peaks of $\Delta n=0$, e.g., $\omega_{\varrho \varkappa}^{11}$ and $\omega_{\varrho \varkappa}^{22}$. When $k_{y}=k_{m i d}$, the symmetry of the wave functions is destroyed. Consequently, the extra peaks are strengthened, and the original peaks are weakened or even disappear (thick dashed line in Fig. 10.20(b)). The corruption of the orthogonality of the sublattices $A_{\mathbf{o}}^{c(v)}$ and $B_{\mathbf{o}}^{v(c)}$ enables the subband transitions to occur from $\Delta n=3$; such examples are seen in $\omega_{\text {mid }}^{03}$ and $\omega_{\text {mid }}^{14}$. The very strong dispersions of LSs also lead to the splitting of the original peaks associated with $k_{\text {mid }}$. This can account for the $\left(\omega_{m i d}^{01}, \omega_{\varrho \varkappa}^{01}\right)$ peaks and the $\left(\omega_{m i d}^{23}, \omega_{\varkappa \varrho}^{23}, \omega_{\varrho \varkappa}^{23}\right)$ peaks in Fig. 10.20(a).

The modulation period strongly affects the magneto-optical spectrum of the LSs, while the modulation effect is less significant with sufficiently larger period. The absorption peaks from $\Delta n=1$ are much higher than those from $\Delta n \neq 1$, as shown by the green line in Fig. $10.20(\mathrm{c})$ for $V_{M E}=0.02 \gamma_{0}$ at a larger period $R=1580$. The transition energies between the valence and conduction LSs at different $k_{y}$ values are almost the same. Thus, the frequencies $\omega_{\varkappa \varrho}^{n n+1}, \omega_{\varrho \varkappa}^{n n+1}$, and $\omega_{m i d}^{n n+1}$ associated with these LS transitions are all the same. Since the symmetry of the wave function does not degrade much, the absorption peaks of $\Delta n=3$ no longer exist. The modulated electric field $\mathbf{E}=-\nabla_{x} V_{M E}(x)=\left(2 \pi V_{M E} / 3 b^{\prime} R_{M E}\right) \sin \left(2 \pi x / 3 b^{\prime} R_{M E}\right) \widehat{x}$ implies that the same value of $V_{M E} / R_{M E}$ produces the same modulation effect. For instance, the absorption spectrum for $R_{M E}=395$ at $V_{M E}=0.005 \gamma_{0}$ (the purple dashed curve in Fig. 10.20(c)) is almost same as that for $R_{M E}=395$ at $V_{M E}=0,0.005 \gamma_{0}$ owing to $V_{M E} / R_{M E}=0.005 / 395=0.02 / 1580$.

We look at the relationship between the absorption frequency and the potential strength more closely. At $V_{M E}=0$, the peaks denoted by the blue symbols in Fig. 10.21 can only appear if they obey the selection rule $\Delta n=1$. After an external modulation potential is applied, peaks from other selection rules $(\Delta n=0$ and $\Delta n=2)$ appear as shown by the black symbols. Under a weak modulated potential $\left(V_{M E}<0.005 \gamma_{0}\right)$, the peak frequency is hardly affected. If the electric potential continues to grow, some peaks start to split, 


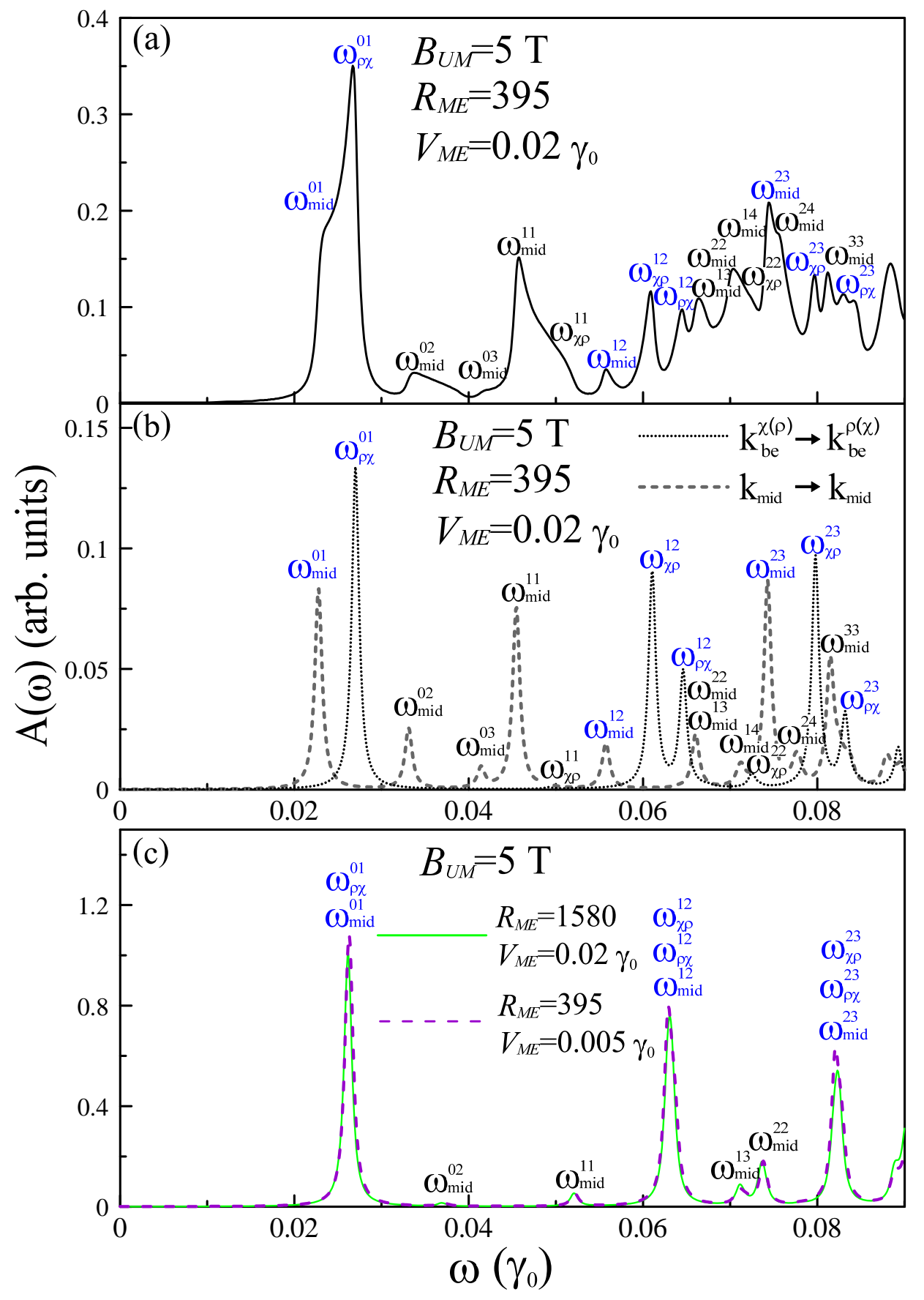

Figure 10.20. The optical absorption spectra for the composite field $B_{U M}=5 \mathrm{~T}$ combined with $R_{M M}=395$ and $V_{M E}=0.02 \gamma_{0}$, where the contributions from the transitions $k_{b e}^{\chi(\rho)} \rightarrow$ $k_{b e}^{\rho(\chi)}$ and $k_{\text {mid }} \rightarrow k_{\text {mid }}$ are shown in (b) by the dotted and dashed curves respectively. (c) A comparison between the absorption spectra for $B_{U M}=5 \mathrm{~T}$ combined with $R_{M M}=395$ and $V_{M E}=0.005 \gamma_{0}$ and $B_{U M}=5 \mathrm{~T}$ combined with $R_{M M}=1580$ and $V_{M E}=0.02 \gamma_{0}$. 
e.g., $\omega_{\varkappa \varrho}^{n n+1}, \omega_{\varrho \varkappa}^{n n+1}$ and $\omega_{m i d}^{n n+1}$. Under a strong potential $\left(V_{M E}>0.02 \gamma_{0}\right)$, more peaks are created by other selection rules, such as $\Delta n=3$ (square markers). Even for the transitions between band-edge states, peaks can be developed by the rule $\Delta n \neq 1$ (solid triangles). Finally, the absorption frequencies related to the $k_{m i d}$ states decrease rapidly with respect to the increment of $V_{M E}$. This is due to the fact that the conduction and valence LSs at $k_{\text {mid }}$ move closer to the Fermi level. These theoretical predictions could be examined by the optical-absorption spectroscopy methods. $76,83,86$ 


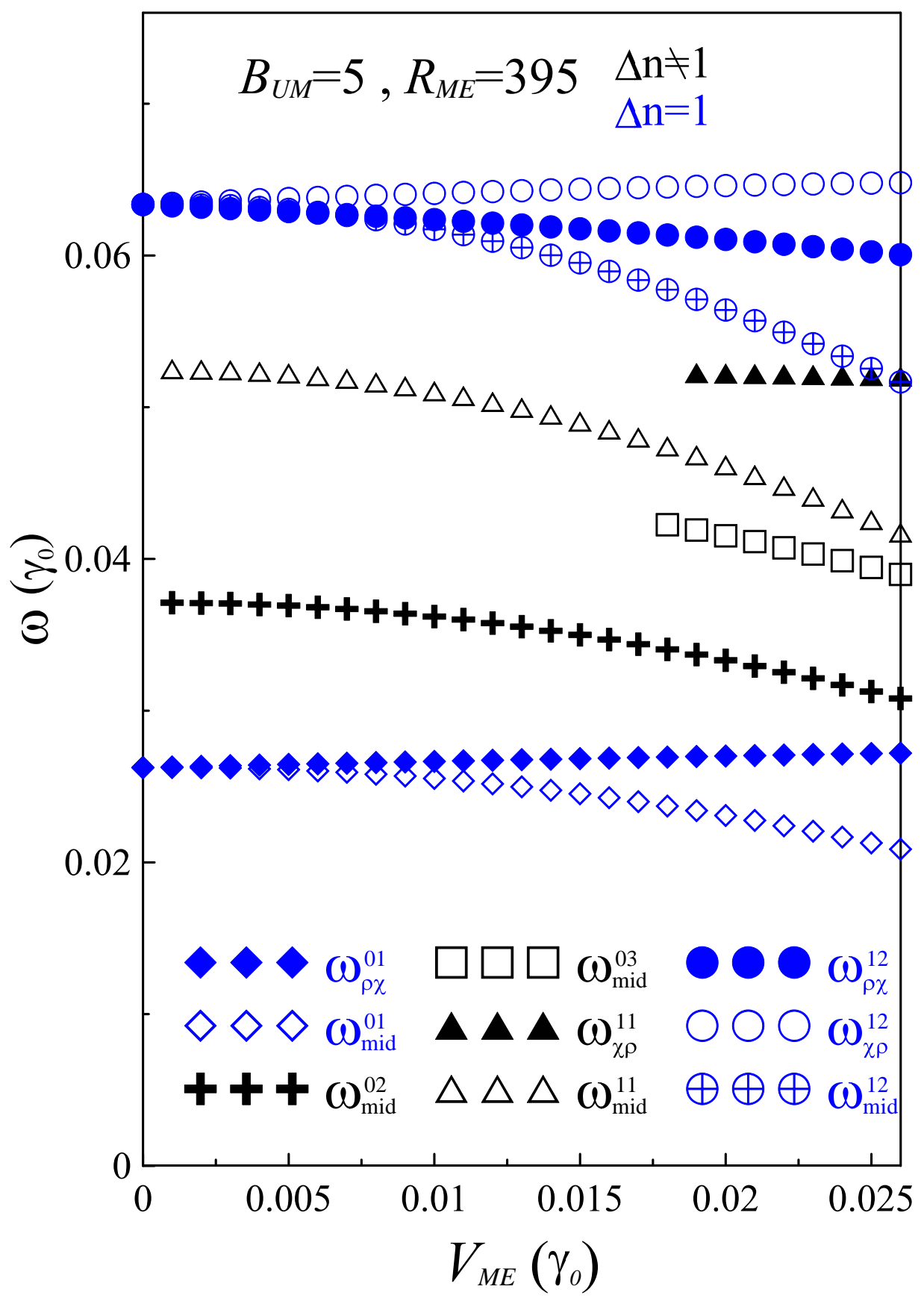

Figure 10.21. The dependence of absorption frequencies, $\omega_{\chi \rho}^{n n^{\prime}}, \omega_{\rho \chi}^{n n^{\prime}}$, and $\omega_{m i d}^{n n^{\prime}}$, on the modulated strength $V_{M E}$. The absorption frequencies with the selection rules $|\Delta n|=$ $\left|n-n^{\prime}\right|=1$ and $|\Delta n| \neq 1$ are indicated by the blue and black colors respectively. 


\section{Conclusion}

The results show that monolayer graphene exhibits the rich optical absorption spectra, an effect being controlled by the external fields. Such fields have a strong influence on the number, intensity, frequency and structure of absorption peaks. Moreover, there would exist the dissimilar selection rules for different external fields. In the presence of the uniform magnetic field, the magneto-optical excitations obey the specific selection rule $\Delta n=1$, since the simple relationship exists between the two sublattices of $a$-and $b$-atoms. As to a modulated magnetic field, an extra selection rule $\Delta n=0$ is obtained, due to the complex overlapping behavior from two subenvelope functions in the wave function. However, the wave functions exhibit irregular behaviors under the modulated electric potential. As a result, it is difficult to single out a particular selection rule. In the composite fields, the symmetry of the LL wave functions is broken by the introduce of two kinds of modulation fields, the modulated magnetic and electric fields, a cause resulting in the altered selection rules in the absorption excitations. The one case is the uniform magnetic field combined with the modulated magnetic field. The extra selection rules, e.g., $\Delta n=2$ and 3 , come to exist when $B_{M M}$ is comparable to $B_{U M}$. Another case is the uniform magnetic field combined with the modulated electric potential. The extra selection rules, e.g., $\Delta n=0,2$ and 3, would be generated in the increase of $V_{M E}$.

For the other graphene systems, the magneto-optical properties corresponding to a perpendicular uniform magnetic field deserve a closer investigation. For example, the AAstacked bilayer graphene is predicted to exhibit two groups of absorption peaks; 72$]$ however, the selection rule $\Delta n=1$ is same as that of MG. As for the AB-stacked bilayer graphene, there exist four groups of absorption peaks and two extra selection rules $(\Delta n=0$ and 2). The few-layer graphenes are expected to display more complex magneto-optical absorption spectra, mainly owing to the number of layers and the stacking configuration.

In this chapter, the generalized tight-binding model is introduced to discuss mono- 
layer graphene under five kinds of external fields. The Hamiltonian, which determines the magneto-electronic properties, is a giant Hermitian matrix for the experimental fields. It is transformed into a band-like matrix by rearranging the tight-binding functions; furthermore, the characteristics of wave function distributions in the sublattices are used to reduce the numerical computation time. In the generalized tight-binding model, the $\pi$-electronic structure of MG is solved in the wide energy range of $\pm 6 \mathrm{eV}$, a solution proving valid even if the magnetic, electric or composite field is applied. Moreover, the important interlayer atomic interactions, not just treated as the perturbations, could be simultaneous included in the calculations. The generalized model can also be extensible to other layer stacked systems, i.e., AA-, AB-, ABC-stacked FLGs 87 91] and bulk graphite. 57, 92, 94 


\section{References}

[1] K. S. Novoselov, A. K. Geim, S. V. Morozov, D. Jiang, Y. Zhang, and S. V. Dubonos, "Electric field effect in atomically thin carbon films." Science 306 (2004): 666.

[2] K. S. Novoselov, A. K. Geim, S. V. Morozov, D. Jiang, M. I. Katsnelson, I. V. Grigorieva, S. V. Dubonos, and A. A. Firsov, "Two-dimensional gas of massless Dirac fermions in graphene." Nature (London) 438, (2005): 197.

[3] C. R. Dean, L. Wang, P. Maher, C. Forsythe, F. Ghahari, Y. Gao, J. Katoch, M. Ishigami, P. Moon, M. Koshino, T. Taniguchi, K. Watanabe, K. L. Shepard, J. Hone, and P. Kim, "Hofstadter's butterfly and the fractal quantum Hall effect in moire superlattices," Nature 497 (2013): 598-602.

[4] G. S. Diniz, M. R. Guassi, and F. Qu, "Engineering the quantum anomalous Hall effect in graphene with uniaxial strains," J. Appl. Phys. 114 (2013): 243701.

[5] S. Gattenloehner, W. R. Hannes, P. M. Ostrovsky, I. V. Gornyi, A. D. Mirlin, and M. Titov, "Quantum Hall Criticality and Localization in Graphene with Short-Range Impurities at the Dirac Point," Phys. Rev. Lett. 112 (2014): 026802.

[6] M. Golor, T. C. Lang, and S. Wessel, "Quantum Monte Carlo studies of edge magnetism in chiral graphene nanoribbons," Phys. Rev. B 87 (2013): 155441.

[7] G. Gumbs, A. Iurov, H. Danhong, P. Fekete, and L. Zhemchuzhna, "Effects of periodic scattering potential on Landau quantization and ballistic transport of electrons in graphene," AIP Conference Proceedings 1590 (2014): 134-142.

[8] B. Jabakhanji, C. Consejo, N. Camara, W. Desrat, P. Godignon, and B. Jouault, "Quantum Hall effect of self-organized graphene monolayers on the C-face of $6 \mathrm{H}$ SiC," J. Phys. D: Appl. Phys. 47 (2014): 094009. 
[9] Y. Kim, K. Choi, J. Ihm, and H. Jin, "Topological domain walls and quantum valley Hall effects in silicene," Phys. Rev. B 89 (2014): 085429.

[10] Y.-X. Wang, F.-X. Li, and Y.-M. Wu, "Quantum Hall effect of Haldane model under magnetic field," Epl 105 (2014): 17002.

[11] Q. Zhenhua, R. Wei, C. Hua, L. Bellaiche, Z. Zhenyu, A. H. MacDonald, and N. Qian, "Quantum Anomalous Hall Effect in Graphene Proximity Coupled to an Antiferromagnetic Insulator," Phys. Rev. Lett. 112 (2014): 116404.

[12] J. H. Ho, Y. H. Lai, Y. H. Chiu, and M. F. Lin, "Landau levels in graphene." Physica E 40 (2008): 1722.

[13] F. D. M. Haldane, "Model for a Quantum Hall Effect without Landau Levels: Condensed-Matter Realization of the "Parity Anomaly"" Phys. Rev. Lett. 61 (1988): 2015.

[14] Z. Jiang, E. A. Henriksen, L. C. Tung, Y. J. Wang, M. E. Schwartz, M. Y. Hun, P. Kim, and H. L. Stormer, "Infrared spectroscopy of Landau levels of graphene." Phys. Rev. Lett. 98 (2007): 197403.

[15] Y. Zhang, Y. W. Tan, H. L. Stormer, and P. Kim, "Experimental observation of the quantum Hall effect and Berry's phase in graphene." Nature 438 (2005): 201.

[16] P. R. Wallace, "The band theory of graphite." Phys. Rev. 71 (1947): 622.

[17] M. I. Katsnelson, K. S. Novoselov, and A. K. Geim, "Chiral tunnelling and the Klein paradox in graphene." Nature Physics 2 (2006): 620.

[18] K. I. Bolotin, F. Ghahari, M. D. Shulman, H. L. Stormer, and P. Kim, "Observation of the fractional quantum Hall effect in graphene." Nature 462 (2009): 196. 
[19] K. S. Novoselov, et al. "Room-temperature quantum Hall effect in graphene." Science 315 (2007): 1379.

[20] R. S. Deacon, K. C. Chuang, R. J. Nicholas, K. S. Novoselov, and A. K. Geim, "Cyclotron resonance study of the electron and hole velocity in graphene monolayers." Phys. Rev. B 76 (2007): 081406.

[21] H. A. Carmona, et al. "Two dimensional electrons in a lateral magnetic superlattice." Phys. Rev. Lett. 74 (1995): 3009.

[22] M. Kato, A. Endo, S. Katsumoto, and Y. Iye, "Two-dimensional electron gas under a spatially modulated magnetic field: A test ground for electron-electron scattering in a controlled environment." Phys. Rev. B 58 (1998): 4876.

[23] A. Messica, A. Soibel, U. Meirav, A. Stern, H. Shtrikman, V. Umansky, and D. Mahalu, "Suppression of conductance in surface superlattices by temperature and electric field." Phys. Rev. Lett. 78 (1997): 705.

[24] C. G. Smith, et al. "Fabrication and physics of lateral superlattices with $40 \mathrm{~nm}$ pitch on high-mobility GaAs GaAlAs heterostructures." J. Vac. Sci. Technol. B 10 (1992): 2904.

[25] A. Soibel, U. Meirav, D. Mahalu, and H. Shtrikman, "Magnetoresistance in a backgated surface superlattice." Phys. Rev. B 55 (1996): 4482.

[26] S. Goswami, et al. "Transport through an electrostatically defined quantum dot lattice in a two-dimensional electron gas." Phys. Rev. B 85 (2012): 075427.

[27] M. Kato, A. Endo, M. Sakairi, S. Katsumoto, and Y. Iye, "Electron-electron Umklapp process in two-dimensional electron gas under a spatially alternating magnetic field." J. Phys.Soc. Jpn. 68 (1999): 1492. 
[28] Y. H. Chiu, J. H. Ho, C. P. Chang, D. S. Chuu, and M. F. Lin, "Low-frequency magneto-optical excitations of a graphene monolayer: Peierls tight-binding model and gradient approximation calculation." Phys. Rev. B 78 (2008): 245411.

[29] Y. H. Chiu, Y. C. Ou, Y. Y. Liao, and M. F. Lin, "Optical-absorption spectra of single-layer graphene in a periodic magnetic field.” J. Vac. Sci. Technol. B 28, (2010) 386-390.

[30] F. Sattari, and E. Faizabadi, "Spin transport through electric field modulated graphene periodic ferromagnetic barriers," Physica B 434 (2014): 69-73.

[31] H. Yan, Z.-D. Chu, W. Yan, M. Liu, L. Meng, M. Yang, Y. Fan, J. Wang, R.-F. Dou, Y. Zhang, Z. Liu, J.-C. Nie, and L. He, "Superlattice Dirac points and spacedependent Fermi velocity in a corrugated graphene monolayer," Phys. Rev. B 87 (2013): 075405 .

[32] L. Zheng-Fang, W. Qing-Ping, X. Xian-Bo, and L. Nian-Hua, "Enhanced magnetoresistance in graphene nanostructure modulated by effective exchange field and Fermi velocity," J. Appl. Phys. 113 (2013): 183704-183704.

[33] J. H. Ho, Y. H. Lai, C. L. Lu, J. S. Hwang, C. P. Chang, and M. F. Lin, ”Electronic structure of a monolayer graphite layer in a modulated electric field", Phys. Lett. A 359 (2006): 70-75.

[34] Y. H. Chiu, J. H. Ho, Y. H. Ho, D. S. Chuu, and M. F. Lin, "Effects of a modulated electric field on the optical absorption spectra in a single-layer graphene", J. Nanosci. Nanotechnol. 9 (2009): 6579-6586.

[35] J. H. Ho, Y. H. Chiu, S. J. Tsai, and and M. F. Lin*, "Semimetallic graphene in a modulated electric potential", Phys. Rev. B 79 (2009): 115427. 
[36] SK Firoz Islam, Naveen K Singh and Tarun Kanti Ghosh, "Thermodynamic properties of a magnetically modulated graphene monolayer." Journal of Physics: Condensed Matter 23 (2011): 445502.

[37] M. Tahir, K. Sabeeh and A. MacKinnon, "Temperature effects on the magnetoplasmon spectrum of a weakly modulated graphene monolayer." Journal of Physics: Condensed Matter 23 (2011): 425304.

[38] M. Tahir, K. Sabeeh and A. MacKinnon, "Weiss oscillations in the electronic structure of modulated graphene." Journal of Physics: Condensed Matter 19 (2007): 406226.

[39] M. Tahir and K. Sabeeh, "Theory of Weiss oscillations in the magnetoplasmon spectrum of Dirac electrons in graphene." Physical Review B 76.19 (2007): 195416.

[40] A. Matulis and F. M. Peeters, "Appearance of enhanced Weiss oscillations in graphene: Theory." Physical Review B 75 (2007): 125429.

[41] Y. C. Ou, J. K. Sheu, Y. H. Chiu, R. B. Chen, and M. F. Lin, "Influence of modulated fields on the Landau level properties of graphene", Phys. Rev. B 83 (2011): 195405.

[42] Y. C. Ou, Y. H. Chiu, J. M. Lu, W. P. Su, and M. F. Lin, "Electric modulation effect on magneto-optical spectrum of monolayer graphene" Comput. Phys. Commun. 184 (2013): 1821-1826.

[43] C. P. Chang, C. L. Lu, F. L. Shyu, R. B. Chen, Y. K. Fang, and M. F. Lin, "Magnetoelectronic properties of a graphite sheet." Carbon 42 (2004): 2975.

[44] A. Gruüneis, et al. "Tight-binding description of the quasiparticle dispersion of graphite and few-layer graphene." Phys. Rev. B 78 (2008): 205425.

[45] J. C. Slonczewski, and P. R. Weiss, "Band Structure of Graphite." Phys. Rev. 109 (1958): 272-279. 
[46] J. C. Charlier, J. P. Michenaud, and X. Gonze, "First-Principles Study of the Electronic Properties of Simple Hexagonal Graphite." Phys. Rev. B 46 (1992): 4531-4539.

[47] S. Latil, and L. Henrard, "Charge Carriers in Few-Layer Graphene Films." Phys. Rev. Lett. 97 (2006): 036803.

[48] B. Partoens, and F. M. Peeters, "From Graphene to Graphite: Electronic Structure around the K Point." Phys. Rev. B 74 (2006): 075404.

[49] C. L. Lu, C. P. Chang, Y. C. Huang, R. B. Chen, and M. L. Lin, "Influence of an Electric Field on the Optical Properties of Few-Layer Graphene with AB Stacking." Phys. Rev. B 73 (2006): 144427.

[50] Y. H. Lai, J. H. Ho, C. P. Chang, and M. F. Lin, "Magnetoelectronic Properties of Bilayer Bernal Graphene." Phys. Rev. B 77 (2008): 085426.

[51] E. McCann, and V. I. Fal'ko, "Landau-Level Degeneracy and Quantum Hall Effect in a Graphite Bilayer." Phys. Rev. Lett. 96 (2006): 086805.

[52] D. S. L. Abergel, and V. I. Fal'ko, "Optical and Magneto-Optical Far-Infrared Properties of Bilayer Graphene.” Phys. Rev. B 75 (2007): 155430.

[53] C. L. Lu, C. P. Chang, Y. C. Huang, J. M. Lu, C. C. Hwang, and M. F. Lin, "Low-energy electronic properties of the AB-stacked few-layer graphites." J. Phys.: Condens. Matter 18 (2006): 5849-5859.

[54] C. L. Lu, C. P. Chang, Y. C. Huang, J. H. Ho, C. C. Hwang, and M. F. Lin, "Electronic properties of AA- and ABC-stacked few-layer graphites." J. Phys. Soc. Jpn. 76 (2007): 024701.

[55] C. L. Lu, H. L. Lin, C. C. Hwang, J. Wang, C. P. Chang, and M. F. Lin, " Absorption spectra of trilayer rhombohedral graphite.” Appl. Phys. Lett. 89 (2006): 221910. 
[56] M. Koshino, and T. Ando, "Magneto-Optical Properties of Multilayer Graphene." Phys. Rev. B 77 (2008): 115313.

[57] R. B. Chen, Y. H. Chiu, and M. F. Lin, "A theoretical evaluation of the magnetooptical properties of AA-stacked graphite." Carbon 54 (2012): 248-276.

[58] M. Koshino, and E. McCann, "Landau level spectra and the quantum Hall effect of multilayer graphene." Phy. Rev. B 83 (2011): 165443.

[59] T. Taychatanapat, K. Watanabe, T. Taniguchi, and P. Jarillo-Herrero, "Quantum Hall effect and Landau-level crossing of Dirac fermions in trilayer graphene." Nature Phys. 7 (2011): 621-625.

[60] C. Faugeras, et al. "Probing the band structure of quadri-layer graphene with magneto-phonon resonance." New J. Phys 14 (2012): 095007.

[61] M. Orlita, et al. "Magneto-optics of bilayer inclusions in multilayered epitaxial graphene on the carbon face of SiC.” Phys. Rev. B 83 (2011): 125302.

[62] N. A. Goncharuk, et al. "Infrared magnetospectroscopy of graphite in tilted fields." Phys. Rev. B 86 (2012): 155409.

[63] Y. H. Chiu, Y. H. Lai, J. H, Ho, D. S. Chuu, and M. F. Lin, "Electronic structure of a two-dimensional graphene monolayer in a spatially modulated magnetic field: Peierls tight-binding model." Phys. Rev. B 77 (2008): 045407.

[64] N. Nemec and G. Cuniberti, "Hofstadter butterflies of bilayer graphene." Phys. Rev. B 75 (2007): 201404(R).

[65] T. G. Pedersen, "Tight-binding theory of Faraday rotation in graphite." Phys. Rev. B 68 (2003): 245104. 
[66] G. Dresselhaus and M. S. Dresselhaus, "Fourier expansion for the electronic energy bands in silicon and germanium." Phys. Rev. 160, (1967): 649.

[67] N. V. Smith, "Photoemission spectra and band structures of d-band metals. VII. Extensions of the combined interpolation scheme." Phys. Rev. B 19, (1979): 50195027.

[68] L. C. Lew Yan Voon, and L. R. Ram-Mohan, "Tight-binding representation of the optical matrix elements: Theory and applications." Phys. Rev. B 47 (1993): 15500.

[69] L. G. Johnson, and G. Dresselhaus, "Optical properies of graphite." Phys. Rev. B 7 (1973): 2275 .

[70] J. Blinowski, et al. "Band structure model and dynamical dielectric function in lowest stages of graphite acceptor compounds." J. Phys. (Paris) 41 (1980): 47-58.

[71] M. F. Lin, and K. W. K. Shung, "Plasmons and optical properties of carbon nanotubes." Phys. Rev. B 50 (1994): 17744.

[72] Y. H. Ho, Y. H. Chiu, D. H. Lin, C. P. Chang, and M. F. Lin, "Magneto-optical selection rules in bilayer Bernal graphene.” ACS Nano 4 (2010): 1465-1472.

[73] Y. H. Ho, J. Y. Wu, R. B. Chen, Y. H. Chiu, and M. F. Lin, "Optical transitions between Landau levels: AA-stacked bilayer graphene.” Appl. Phys. Lett. 97 (2010): 101905.

[74] Y. Zheng and T. Ando, "Hall conductivity of a two-dimensional graphite system." Phys. Rev. B 65 (2002): 245420.

[75] M. Orltia, et al. "Graphite from the viewpoint of Landau level spectroscopy: An effective graphene bilayer and monolayer." Phys Rev Lett 102 (2009): 166401. 
[76] J. M. Dawlaty, et al. "Measurement of the optical absorption spectra of epitaxial graphene from terahertz to visible." Appl Phys Lett 93 (2008): 131905.

[77] K.-C. Chuang, A. M. R. Baker, and R. J. Nicholas, "Magnetoabsorption study of Landau levels in graphite." Phys. Rev. B 80 (2009): 161410(R).

[78] M. Orlita, C. Faugeras, G. Martinez, D. K. Maude, M. L. Sadowski, and M. Potemski, "Dirac fermions at the $\mathrm{H}$ point of graphite: magnetotransmission studies." Phys. Rev. Lett. 100 (2008): 136403.

[79] M. L. Sadowski, G. Martinez, M. Potemski, C. Berger, and W. A. de Heer, "Landau level spectroscopy of ultrathin graphite layers." Phys. Rev. Lett. 97 (2006): 266405.

[80] K. S. Novoselov, et al. "Unconventional quantum Hall effect and Berry's phase of $2 \pi$ in bilayer graphene." Nat. Phys. 2 (2006): 177.

[81] A. J. M. Giesbers, U. Zeitler, M. I. Katsnelson, L. A. Ponomarenko, T. M. Mohiuddin, and J. C. Maan, "Quantum-Hall activation gaps in graphene." Phys. Rev. Lett. 99 (2007): 206803.

[82] J. G. Checkelsky, L. Li, and N. P. Ong, "Zero-energy state in graphene in a high magnetic field." Phys. Rev. Lett. 100 (2008): 206801.

[83] S. Thongrattanasiri, F. H. L. Koppens, and F. J. García de Abajo, "Complete optical absorption in periodically patterned graphene." Phys. Rev. Lett. 108 (2012): 047401.

[84] V. G. Kravets, et al. "Spectroscopic ellipsometry of graphene and an exciton-shifted van Hove peak in absorption." Phys. Rev. B 81 (2010): 155413.

[85] M. Orlita and M. Potemski, "Dirac electronic states in graphene systems: optical spectroscopy studies." Semicond. Sci. Technol. 25 (2010): 063001. 
[86] F. Wang, et al. "Gate-variable optical transitions in graphene." Science 320 (2008): 206.

[87] Y. C. Chuang, J. Y. Wu, and M. F. Lin, "Electric Field Dependence of Excitation Spectra in AB-Stacked Bilayer Graphene.” Scientific Reports 3 (2013): 1368.

[88] Y. H. Ho, S. J. Tsai, M. F. Lin, and W. P. Su, "Unusual Landau levels in biased bilayer Bernal graphene.” Phys. Rev. B 87 (2013): 075417.

[89] H. C. Chung, W. P. Su, and M. F. Lin, "Electric-field-induced destruction of quasiLandau levels in bilayergraphene nanoribbons." Phys. Chem. Chem. Phys, 15 (2012): $868-875$.

[90] M. F. Lin, Y. C. Chuang, and J. Y. Wu, "Electrically tunable plasma excitations in AA-stacked multilayer graphene." Phys. Rev. B 86 (2012): 125434.

[91] C. W. Chiu, Y. C. Huang, F. L. Shyu, and M. F. Lin, "Optical absorption spectra in ABC-stacked graphene superlattice." Synthetic Metals 162 (2012): 800-804.

[92] C. H. Ho, C. P. Chang, W. P. Su and M. F. Lin, "Processing anisotropic Dirac cone and Landau subbands along a nodal spiral." New J. Phys. 15 (2013): 053032.

[93] C. W. Chiu, F. L. Shyu, M. F. Lin, Godfrey Gumbs, and Oleksiy Roslyak, "Anisotropy of $\pi$-plasmon Dispersion Relation of AA-stacked Graphite." J. Phys. Soc. Jpn. 81 (2012): 104703.

[94] C. H. Ho, Y. H. Ho, Y. Y. Liao, Y. H. Chiu, C. P. Chang, and M. F. Lin, "Diagonalization of Landau Level Spectra in Rhombohedral Graphite." J. Phys. Soc. Jpn. 81 (2012): 024701 . 


\section{Figure Captions}

Fig. 10.1 The primitive unit cell of monolayer graphene (a) in the absence and (b) in the presence of external fields.

Fig. 10.2 (a) The Landau level spectrum for the uniform magnetic field $B_{U M}=5 \mathrm{~T}$. The Landau level wave functions corresponding to (b) the $a$ - and (c) the $b$-atoms.

Fig. 10.3 The optical absorption spectra for (a) $B_{U M}=5 \mathrm{~T}$ and (b) $B_{U M}=10 \mathrm{~T}$. (c) The dependence of the absorption frequency on the square root of field strength $B_{U M}$.

Fig. 10.4 The energy dispersions and the illustration of optical excitation channels for the modulated magnetic field along the armchair direction with $R_{M M}=500$ and $B_{M M}=10$ T.

Fig. 10.5 The wave functions of Quasi-Landau levels at (a)-(f) the original band-edge state $k_{1}$ with the quantum numbers $n^{c, v}=0, n^{c}=1$ and $n^{c}=2$, (g) and (h) the split point $k_{2}$ with $n^{c}=1$, and (i)-(l) two extra band-edge states $k_{3}$ and $k_{4}$ with $n^{c}=1$.

Fig. 10.6 The optical absorption spectra for (a) $B_{M M}=10 \mathrm{~T}$ at a fixed periodic length with modulation and polarization along the armchair and zigzag directions and (b) different modulation periods and field strengths with both the modulation and polarization along the armchair direction.

Fig. 10.7 The dependence of the absorption frequency on (a) the period $R_{M M}$ and (b) the square root of field strength $B_{M M}$.

Fig. 10.8 The energy dispersions for the modulated electric potential along the armchair direction with $R_{M M}=500$ and $V_{M E}=0.05 \gamma_{0}$.

Fig. 10.9 The optical absorption spectra corresponding to Fig. 10.8, which includes the 
contributions from the $\mu$ and $\nu$ states, respectively.

Fig. 10.10 The optical absorption for (a) $V_{M E}=0.05 \gamma_{0}$ at a fixed periodic length with modulation and polarization along the armchair and zigzag directions and (b) different modulation periods and field strengths with both the modulation and polarization along the armchair direction.

Fig. 10.11 The wave functions at different band-edge states, the $\mu_{i}^{c}$ and $\nu_{i}^{c}$ states for $i=1^{\sim} 7$.

Fig. 10.12 The energy dispersions for (a) the uniform magnetic field $B_{U M}=5 \mathrm{~T}$ by the red curves and the composite field $B_{U M}=5 \mathrm{~T}$ combined with $R_{M M}=395$ and $B_{M M}=1$ T by the black curves, (b) $B_{U M}=5 \mathrm{~T}$ combined with $R_{M M}=395$ and $B_{M M}=5 \mathrm{~T}$, and (c) $B_{U M}=5 \mathrm{~T}$ combined with $R_{M M}=395$ and $B_{M M}=40 \mathrm{~T}$. All modulated fields are applied along the armchair direction.

Fig. 10.13 The wave functions with $n^{c, v}=0$ and $n^{c}=1$ at $k_{b e}^{\zeta}$ for (a)-(d) the uniform magnetic field $B_{U M}=5 \mathrm{~T}$ by the red curves and the composite field $B_{U M}=5 \mathrm{~T}$ combined with $R_{M M}=395$ and $B_{M M}=1 \mathrm{~T}$ by the black curves, (e)-(h) $B_{U M}=5 \mathrm{~T}$ by the red curves and $B_{U M}=5 \mathrm{~T}$ combined with $R_{M M}=395$ and $B_{M M}=5 \mathrm{~T}$ by the black curves, and (i)-(l) $B_{U M}=5 \mathrm{~T}$ combined with $R_{M M}=395$ and $B_{M M}=40 \mathrm{~T}$.

Fig. 10.14 The optical absorption spectra corresponding to (a) the uniform magnetic field $B_{U M}=5 \mathrm{~T}$ by the red curve and the composite field $B_{U M}=5 \mathrm{~T}$ combined with $R_{M M}=395$ and $B_{M M}=1 \mathrm{~T}$ by the black curve and (b) the composite field $B_{U M}=5 \mathrm{~T}$ combined with $R_{M M}=395$ and $B_{M M}=5 \mathrm{~T}$.

Fig. 10.15 The optical absorption spectra corresponding to the composite field $B_{U M}=5$ T combined with $R_{M M}=395$ and $B_{M M}=40 \mathrm{~T}$ by the red dotted curve and the pure 
modulated magnetic field $R_{M M}=395$ and $B_{M M}=40$ T by the black curve.

Fig. 10.16 The dependence of absorption frequencies, $\omega_{\eta}^{n n^{\prime}}$ and $\omega_{\zeta}^{n n^{\prime}}$ with $|\Delta n|=$ $\left|n-n^{\prime}\right|=1$, on the modulated strength $B_{M M}$.

Fig. 10.17 The energy dispersions for (a) the uniform magnetic field $B_{U M}=5 \mathrm{~T}$ by the red curves and the composite field $B_{U M}=5 \mathrm{~T}$ combined with $R_{M M}=395$ and $V_{M E}=0.005 \gamma_{0}$ by the black curves, (b) $B_{U M}=5 \mathrm{~T}$ combined with $R_{M M}=395$ and $V_{M E}=0.02 \gamma_{0}$. All modulated fields are applied along the armchair direction.

Fig. 10.18 The wave functions for (a)-(d) $n^{c, v}=0, n^{c}=1, n^{c}=5$, and $n^{v}=5$ at $k_{m i d}$, (i)-(n) $n^{c, v}=0, n^{c}=1$ and $n^{c}=5$ at $k_{b e}^{\rho}$, and $(\mathrm{l})-(\mathrm{m}) n^{v}=5$ at $k_{b e}^{\chi}$. The results corresponding to the uniform magnetic field $B_{U M}=5 \mathrm{~T}$, the composite field $B_{U M}=5$ T combined with $R_{M M}=395$ and $V_{M E}=0.005 \gamma_{0}$, and $B_{U M}=5 \mathrm{~T}$ combined with $R_{M M}=395$ and $V_{M E}=0.02 \gamma_{0}$ are indicated by the red, black, and blue curves respectively.

Fig. 10.19 The optical absorption spectra corresponding to (a) the uniform magnetic field $B_{U M}=5 \mathrm{~T}$ by the red curve and the composite field $B_{U M}=5 \mathrm{~T}$ combined with $R_{M M}=395$ and $V_{M E}=0.005 \gamma_{0}$ by the black curve, $(\mathrm{b})$ the transitions of $k_{b e}^{\chi(\rho)} \rightarrow k_{b e}^{\rho(\chi)}$ and $k_{\text {mid }} \rightarrow k_{\text {mid }}$ for $B_{U M}=5 \mathrm{~T}$ combined with $R_{M M}=395$ and $V_{M E}=0.005 \gamma_{0}$ by the dotted and dashed curves respectively, and (c) the higher frequency region of $0.1^{\sim} 0.2 \gamma_{0}$.

Fig. 10.20 (a) The optical absorption spectra for the composite field $B_{U M}=5 \mathrm{~T}$ combined with $R_{M M}=395$ and $V_{M E}=0.02 \gamma_{0}$, where the contributions from the transitions $k_{b e}^{\chi(\rho)} \rightarrow k_{b e}^{\rho(\chi)}$ and $k_{m i d} \rightarrow k_{m i d}$ are shown in (b) by the dotted and dashed curves respectively. (c) A comparison between the absorption spectra for $B_{U M}=5 \mathrm{~T}$ combined with $R_{M M}=395$ and $V_{M E}=0.005 \gamma_{0}$ and $B_{U M}=5 \mathrm{~T}$ combined with $R_{M M}=1580$ and $V_{M E}=0.02 \gamma_{0}$. 
Fig. 10.21 The dependence of absorption frequencies, $\omega_{\chi \rho}^{n n^{\prime}}, \omega_{\rho \chi}^{n n^{\prime}}$, and $\omega_{m i d}^{n n^{\prime}}$, on the modulated strength $V_{M E}$. The absorption frequencies with the selection rules $|\Delta n|=$ $\left|n-n^{\prime}\right|=1$ and $|\Delta n| \neq 1$ are indicated by the blue and black colors respectively. 